\title{
Appraisal of role of the polyanionic inducer length on amyloid formation by 412-residue 1N4R Tau protein: A comparative study
}

\author{
Abolfazl Jangholi1,2, Mohammad Reza Ashrafi-Kooshk', Seyed Shahriar Arab ${ }^{3}$, \\ Gholamhossein Riazi ${ }^{4}$, Farzad Mokhtari, Mansour Poorebrahim5, Hamid Mahdiuni², Boris \\ I. Kurganov6, Ali Akbar Moosavi-Movahedi7, Reza Khodarahmi*1,8
}

${ }^{1}$ Medical Biology Research Center, Kermanshah University of Medical Sciences, Kermanshah, Iran

2 Department of Biology, Faculty of Science, Razi University, Kermanshah, Iran

${ }^{3}$ Department of Biophysics, Faculty of Biological Sciences, Tarbiat Modares University, Tehran, Iran

${ }^{4}$ Institute of Biochemistry and Biophysics, University of Tehran, Tehran, Iran

${ }^{5}$ Department of Medical Biotechnology, School of Advanced Technologies in Medicine, Tehran University of Medical Sciences, Tehran, Iran

${ }^{6}$ Bach Institute of Biochemistry, Research Center of Biotechnology of the Russian Academy of Sciences, 33, bld. 2 Leninsky Ave., Moscow 119071, Russia

${ }^{7}$ Institute of Biochemistry and Biophysics, University of Tehran, Tehran, Iran

${ }^{8}$ Department of Pharmacognosy and Biotechnology, Faculty of Pharmacy, Kermanshah University of Medical Sciences, Kermanshah, Iran

*Corresponding Author: R. Khodarahmi; E-mail: rkhodarahmi@mbrc.ac.ir, rkhodarahmi@kums.ac.ir, Medical Biology Research Center, Kermanshah University of Medical Sciences, Kermanshah, Iran

Tel: 0098-831-4276473

Fax: 0098-831-4276471 


\section{Abstract}

In many neurodegenerative diseases, formation of protein fibrillar aggregates has been observed as a major pathological change. Neurofibrillary tangles, mainly composed of fibrils formed by the microtubule-associated protein; Tau, are a hallmark of a group of neurodegenerative diseases such as Alzheimer's disease. Tau belongs to the class of natively unfolded proteins and partially folds into an ordered $\beta$-structure during aggregation. Polyanionic cofactors such as heparin are commonly used as inducer of Tau aggregation in vitro. The role of heparin in nucleation and elongation steps during Tau fibril formation is not fully understood. In the current study, aggregation kinetics as well as structure of Tau amyloid fibrils, by using the 1N4R isoform, have been reproducibly determined in the presence of heparin and the shorter molecule; enoxaparin. The kinetic studies demonstrated that heparin (not enoxaparin) efficiently accelerates Tau amyloid formation and revealed, mechanistically, that the molecular weight of the inducer is important in accelerating amyloidogenesis. The kinetic parameter values of Tau amyloid aggregation, especially, the amyloid aggregation extent, were relatively different in the presence of heparin and enoxaparin, at various stoichiometries of the inducers binding. Also, based on the results, obtained from CD, FTIR, AFM and XRD studies, it may be suggested that the inducer length plays a critical role mainly in the nucleation process, so that it determines that oligomers lie on or off the pathway of Tau fibrillization. The biochemical results herein suggest that the chemical environment of the extracellular matrix as well as localization of distinct glycosaminoglycans may influence deposition behavior of Tau amyloidosis.

Key words: Heparin, Enoxaparin, Amyloid aggregation, 1N4R Tau isoform 


\section{Introduction}

Amyloid fibrils refers to the insoluble fibers that formed by self-assembling of normally soluble proteins and are resistant to bio-degradation. Their formation can lead to disease such as Alzheimer's disease, Parkinson`s disease, Diabetes type 2 and the spongiform encephalopathies that each disease is characterized by a specific protein or peptide that aggregates. The fibrils are inherently stable and structural studies have revealed that they are composed predominantly of $\beta$-sheet structure in a characteristic cross- $\beta$ conformation [1,2]. Alzheimer's disease (AD) is characterized by presence of two pathological hallmarks, extracellular senile plaques and intracellular neurofibrillary tangles (NFTs) [3]. Senile plaques formed primarily by the amyloid beta peptide $(A \beta)$ [4]. Tauopathies are also a group of neurodegenerative diseases characterized by the aggregation of Tau protein into filamentous lesions such as NFTs. Tau protein, which is main component of NFTs, belongs to the family of microtubule-associated protein (MAP) is an essential factor of the neuronal cytoskeleton and is involved in kinesin-dependent axonal transport, microtubule stabilization and neurite outgrowth [5,6] and exists as six alternatively spliced isoforms, arising from a single gene located on chromosome 17 [7]. This intrinsically disordered protein (IDP), rich in charged amino acid residues, characterized by its low content of secondary structure (see Fig. 1), its sequence is dominated by hydrophilic residues and a high level of conformational flexibility, consistent with its resistance to heat and acid treatment [8-10]. Isoforms differ from one another by the presence or absence of a 29 or 58 amino acid insert in the $\mathrm{N}$-terminal half $(0,1$ and $2 \mathrm{~N})$ and by the inclusion, or exclusion, of a 31 amino acid repeat, encoded by exon 10 of Tau, in the C-terminal half of the protein [7]. These highly conserved repeats constitute the microtubule proteins (MTP) binding region of Tau; therefore C-terminal part of Tau encompasses either three or four (3R and 4R) contiguous microtubule binding repeats (MTBRs) (Fig. 1) [7]. It has been found that these isoforms stabilize MTP both in vitro and in vivo and $4 \mathrm{R}$ Tau stabilizes MTP more strongly and also overexpresses in $\mathrm{AD}$ condition compared to $3 \mathrm{R}$ isoform [11]. In this way, the positive lysine residues in the MTBRs of Tau interact with the negative C-terminal of tubulin in a sequence-specific fashion [12].

Because $\mathrm{AD}$ is the most common of tauopathologies, studies of the pathological process of Tau aggregation are of great interest. Hyperphosphorylated forms of Tau fibrils, as the major protein 
components of the NFTs observed in AD, [13] and the amount of these fibrillar aggregates correlates with cytoskeletal disorganization, neuronal death and finally cognitive impairment.

$\mathrm{AD}$ is primarily a sporadic disease with a significant environmental contribution to relative risk. Therefore, the mechanisms through which Tau fibrillizes may provide clues to the initiating stresses that precede neurodegeneration. However, Tau is a highly water-soluble protein that polymerizes weakly at physiological concentrations, so that detailed characterization of the Tau fibrillization reaction has been hampered by the failure of full-length Tau proteins to spontaneously aggregate over experimentally tractable time periods. This limitation has been overcome by the addition of exogenous fibrillization promoters, so that, inspired from pathogenic in vivo Tau hyperphosphorylation. Amyloidogenesis process of the protein can be triggered/accelerated by polyanionic inducers such as glycosaminoglycan heparin, whose structure closely mimics the highly sulfated regions of heparan sulfate (HS). Additionally, it has been shown that Tau NFTs in the brains of Alzheimer's patients contained the natural polyanion sulfoglycosaminoglycan polymer; HS [14-16]. However, Tau fibrils formed in vitro from recombinant full-length human Tau protein, including assembled filaments induced by the addition of heparin, closely resemble those aggregates isolated from AD-affected brain [6].

Dependency of in vitro aggregation of Tau into $\beta$-sheet-rich fibrils on adding of polyanionic cofactors $[9,17]$ appears to support heterogeneous Tau nucleation (i.e. the foreign anionic substance facilitates filament nucleation on its surface) [18], inspiring that length (MW) of inducer may play a critical role. Moreover, anionic inducers also facilitate seeding reactions [18], suggesting they can also promote conformational changes and stabilize aggregation-prone protein states in full length Tau protein.

Basically, when full-length Tau protein does not polymerize/aggregate, in the absence of point mutations and/or macromolecular inducers, it means that the intrinsic or extrinsic inhibitory factors are existent. Without phosphorylation, the positively charged regions flanking the microtubule binding repeat (MTBR) were proposed to inhibit Tau assembly, where hyperphosphorylation neutralizes these basic inhibitory domains, induce assembly of competent conformations, perhaps associated with enriched $\beta$-sheet content, and enabling Tau-Tau interactions and homogeneous nucleation [19] (see also Fig. 5 in [20]). It has been described that 
heparin interaction both with peptides in the second and third repeats (R2 and R3) and with the basic regions flanking the MTBR, through structural changes in the former and charge neutralization followed by an overall structural change in the latter, enables aggregation of Tau into mature Tau filament or paired helical filament (PHF) [21]. It is noteworthy that Tau molecules, termed "protomers" as subunits of filaments, generally adopt the parallel, in register cross- $\beta$-sheet structure typical of amyloid aggregates and PHFs consist of two protofilaments wound around each other [18]. For PHF aggregation two hexapeptide motifs at the beginning of the R2 and R3 (275VQIINK ${ }^{280}$ and ${ }^{306} V_{Q} I_{V Y K}{ }^{311}$ ) in the largest Tau isoform are crucial [22].

\section{Fig. 1}

Furthermore, exact molecular mechanism of heparin-induced Tau fibril formation is not fully understood because there is still debate about whether heparin plays a role only in the nucleation step or is either required in both nucleation and elongation steps of Tau fibril formation in vitro $[21,23,24]$.

In this study, the 1N4R Tau isoform with maximum similarity to full-length Tau (see Figs. 1 and 2 and the captions) was purified, then we used heparin (average molecular mass of $15000 \mathrm{Da}$ ) and enoxaparin (shorter heparin with molecular mass of $4500 \mathrm{Da}$ ) to test/compare the capacity of these inducers to promote aggregation of 1N4R Tau isoform under near physiological buffer conditions plus reducing environments and to obtain further insight about the effect of inducer length (or inducer MW) on the kinetic of Tau fibril formation. Additionally, as stated earlier, the polyanionic heparin inducer both promotes Tau conformational changes and facilitates the protein filament nucleation on its surface [18](and references 6, 11, 12, and 14 therein). We therefor set out to determine whether the inducer length could affect different aggregation kinetic parameters such as aggregation rate constant $\left(k_{\text {agg }}\right)$, order, extent and nucleation time as well as mechanism and rate-limiting step of aggregation reaction. Actually, this is the first study to demonstrate, quantitatively/mechanistically, the kinetic role of polyanionic inducer length in the Tau amyloid aggregation. The kinetics of fibril formation by Tau protein, in the presence of inducers, has been registered using Thioflavin T (ThT) fluorescence signal. We further analyzed Tau aggregation using several biophysical methods, such as ANS fluorescence, SDS-PAGE, Far-UV CD spectra, X-ray diffraction and atomic force microscopy (AFM). 


\section{Materials and methods}

\subsection{Materials}

Resin of Ni-NTA superflow agarose for efficient purification of His-tagged proteins were obtained from QIAGEN. Thioflavin T (ThT) were purchased from Sigma-Aldrich (Munich, Germany). Heparin (average molecular mass of $15000 \mathrm{Da}$ ) and Enoxaparin (average molecular mass of 4500 $\mathrm{Da}$ ) were purchased from Celsus Laboratories (Cincinnati, OH). 1-Anilinonaphthalene-8-sulfonate (ANS) was purchased from Merck (Darmstadt, Germany). Peptide analogue of PHF6 (VQIVYK) was purchased from Bio-basic Inc. (Canada) .All other chemicals were of analytical grade and used as obtained from the suppliers. All solutions were prepared with double distilled water. A stock solution of ThT and ANS were freshly prepared in Tris-HCl pH 7.6 buffer and stored in dark, at $4{ }^{\circ} \mathrm{C}$.

\subsection{Protein expression and purification}

The His-tagged 1N4R Tau cDNA was inserted into a pET-21a plasmid vector using NdeI/XhoI, and transformed into E.coli BL21 (DE3) for expression. Briefly, bacteria were grown at $37{ }^{\circ} \mathrm{C}$ in $20 \mathrm{~mL}$ of $\mathrm{LB}, 100 \mu \mathrm{g} / \mathrm{mL}$ ampicillin culture for $14 \mathrm{~h}$ before inoculation into a $2 \mathrm{~L}$ culture. After induction with $1 \mathrm{mM}$ IPTG at $37{ }^{\circ} \mathrm{C}$ for $3 \mathrm{~h}$ the harvested cells were resuspended in lysis buffer (50 mM Tris- $\mathrm{HCl}, 100$ mM NaCl, 30 mM Imidazole, 1mM EDTA, 5 mM DTT, 2 mM PMSF, pH 8), and then broken by sonication (Eurosonic 4D, Italy) on ice. After centrifugation which was boiled for $10 \mathrm{~min}$ and the filtered supernatants were loaded onto a Ni-NTA column. The column was washed five times with $50 \mathrm{mM}$ Tris- $\mathrm{HCl}, 500 \mathrm{mM} \mathrm{NaCl}, 30 \mathrm{mM}$ imidazole, pH 8.0, and Tau proteins were eluted with $50 \mathrm{mM}$ Tris- $\mathrm{HCl}, 100 \mathrm{mM} \mathrm{NaCl}, 250 \mathrm{mM}$ imidazole, pH 8.0. The purified protein is 412 amino acids long, with maximum similarity to the longest isoform of Tau (see Fig. 2), has a His tag at the C-terminus. Protein concentration was determined according to the Lowry`s method [25] and standard curve was generated using BSA. The protein purity was confirmed (>98\%) by Coomassie Brilliant Blue staining of 10\% SDS-PAGE.

\subsection{Tau Aggregate Preparation}

It is to be noted that constructs with and without a His-tag have been used in previous studies of the aggregation of the repeat domain as well as full-length Tau, and it was reported that the aggregation mechanism is not affected by the presence of the His tag [6]. In all experiments, aggregation of Tau was prepared containing 16-95 $\mu \mathrm{M}$ Tau, $5 \mathrm{mM}$ DTT , $50 \mathrm{mM}$ Tris-HCl pH 7.6 at 
$37^{\circ} \mathrm{C}$. Protein solutions were induced by desired amounts of Heparin or Enoxaparin. At different indicated time points of aggregation 15-75 $\mu \mathrm{L}$ aliquots of the sample depending on the protein concentration were withdrawn for analysis by ThT \& ANS fluorescence and SDS-PAGE. The total volume of the aggregation protein solution was $1 \mathrm{ml}$. Importantly, the kinetics of Tau aggregation in Tris buffer is highly reproducible, as seen in the small spread in the data (and in [6]) even across different (protein and or) inducer preparations. Additionally, this high reproducibility of the data is because of the care taken to ensure that the starting preparation was free of seeds and was as homogeneous as possible. The seed-free Tau preparations, without heparin induction, displayed no measurable ThT fluorescence signal both at $\mathrm{t}=0$ and $\mathrm{t}=96 \mathrm{~h}$ (data not shown).

\subsection{Gel Electrophoresis}

A $40 \mu \mathrm{L}$ of $58 \mu \mathrm{M}$ aggregation protein solutions were mixed with sample buffer ( $50 \mathrm{mM}$ Tris- $\mathrm{HCl}$ pH 6.8; $2 \%$ SDS; $0.1 \%$ bromophenol blue; $10 \%$ glycerol) and boiled for 5 minutes. The samples were separated on a 10\% SDS-PAGE and stained with Coomassie Brilliant Blue.

\subsection{ThT Fluorescence Assay}

To investigate kinetically whether Tau, in the presence of polyanionic inducer, was converted to amyloid-like fibrils, and to evaluate the inducer length effects on this process, a ThT-based fluorimetric method was employed. All fluorescence measurements were carried out in the ratio mode on a luminescence spectrophotometer at room temperature or as it is stated. ThT assay, as most commonly used to assess Tau assembly in real time, shows enhanced fluorescence at 482 $\mathrm{nm}$ when bound to amyloid fibrils. For ThT assay, a final concentration of $3 \mu \mathrm{M}$ protein and 20 $\mu \mathrm{M}$ ThT was used. For every reading a calculated amount of the protein according to the concentration used in the aggregation experiment was withdrawn from the sample and diluted to $400 \mu \mathrm{L}$ with Tris-HCl pH 7.6. Then ThT fluorescence was measured in a Cary Eclipse (Varian) spectrofluorimeter at $25{ }^{\circ} \mathrm{C}$, with 440 and $482 \mathrm{~nm}$ of excitation and emission wavelengths, respectively (slit width $10 \mathrm{~nm}$ each).

\subsection{ANS Fluorescence Assay}

For ANS assay a final concentration of $3 \mu \mathrm{M}$ protein and $100 \mu \mathrm{M}$ ANS was used. For every reading a calculated amount of the protein according to the concentration used in the aggregation 
experiment was withdrawn from the sample and diluted to $400 \mu \mathrm{L}$ with Tris- $\mathrm{HCl} \mathrm{pH} \mathrm{7.6.} \mathrm{Then}$ ANS fluorescence was measured in a Cary Eclipse (varian) spectrofluorimeter at $25{ }^{\circ} \mathrm{C}$, with 380 and $490 \mathrm{~nm}$ of excitation and emission wavelength respectively (slit width $10 \mathrm{~nm}$ each).

\subsection{Data Analyses}

Plots of blank-subtracted fluorescence values versus time were analyzed with a procedure of best fit, using the following sigmoidal function:

$$
F=\frac{F_{\max }}{1+\exp \left\{-\left[\left(t-t_{\mathrm{m}}\right) / \tau\right]\right\}}
$$

Equation 1

Where $F$ and $F_{\max }$ are the fluorescence intensities at time $t$ and final, respectively, $t_{\mathrm{m}}$ is the time

to formation of half-amounts of aggregates, and $\tau$ is a characteristic time constant. $F_{\max }$ and $\tau$ were used as floating parameters in the procedure of best fit. Thus, the apparent rate constant ( $k_{\text {app }}$ ) for the elongation of fibrils is given by $1 / \tau$ and the lag time $\left(t_{\mathrm{D}}\right)$ was calculated using the formula $t_{\mathrm{D}}=t_{\mathrm{m}}-2 \tau[26,27]$.

\subsection{Far-UV Circular Dichroism}

Changes in secondary structure of samples were analyzed by circular dichroism (CD) spectroscopy. All measurements were carried out with a Jasco J-810 CD spectrometer (Lakewood, NJ, USA), with a $0.1 \mathrm{~cm}$ path length sample cell. The calibration of the instrument was achieved using aqueous solutions of d-10-camphorsulfonic acid (ammonium salt) prior to use [28]. All CD measurements were carried out at $25{ }^{\circ} \mathrm{C}$ with the help of a thermostatically controlled cell holder. Spectra were collected at $1 \mathrm{~nm}$ intervals, with a scan speed of $20 \mathrm{~nm} / \mathrm{min}$. Each spectrum was the average of two scans and noise in the data was smoothed using the instrument software. The final protein concentration was set to $58 \mu \mathrm{M}$. All CD spectra were corrected for background absorbance using a reference sample containing the inducers and buffer. 


\subsection{Atomic Force Microscopy}

For AFM images a $10 \mu \mathrm{L}$ aliquot of the sample that incubated for $200 \mathrm{~h}$ was immediately deposited on freshly cleaved mica and allowed to incubate for $5 \mathrm{~min}$. The mica surface was then washed twice with filtered MiliQ water for $30 \mathrm{~s}$ and dried under a vacuum. AFM images were obtained on a veeco (auto probe cp_research) AFM instrument operating in the non-contact mode using $250 \mathrm{kHz}, 15$ newton/m cantilevers with a rounding tip radius of $10 \mathrm{~nm}$.

\subsection{FTIR experiments}

FTIR experiments were performed at room temperature on a Shimadzu FTIR spectrometer (Japan) equipped with a Germanium attenuated total reflection (ATR) accessory and a DLaTGS detector. All spectra were taken via the ATR approach with resolution of $2 \mathrm{~cm}^{-1}$ and 128 spectra averaged. In the first step, the spectra of Tau solutions in the presence of $16 \mu \mathrm{M}$ heparin and 63 $\mu \mathrm{M}$ enoxaparin were collected as backgrounds. Then the aggregation protein solutions that incubated for $200 \mathrm{~h}$ was recorded under the same conditions.

\subsection{X-Ray Diffraction (XRD) Study}

The samples for XRD, $50 \mu \mathrm{L}$ aliquots of the protein and aggregation solutions, were transferred on glass slides and dried under air. The studies were carried out using a Rigaku X-ray powder diffractometer with $\mathrm{Cu}$ anode ( $\mathrm{Cu}-\mathrm{K} \alpha$ radiation $\lambda=1.54186 \AA)$. The samples were scanned from $5^{\circ}$ to $60^{\circ}$ at $30 \mathrm{kV}$.

\subsection{Computational studies}

An initial PDB file for the 'native' Tau structure was selected from the ensemble of structures generated in previous work by the Zweckstetter group [11] based on the Pearson coefficient of a comparison between HDX data provided by [20] and calculated solvent accessibility. Molecular dynamics (MD) simulations of the native Tau protein was performed in GROMACS version 5 for a period of $40 \mathrm{~ns}$ using the all-atom OPLS force field [29]. Tau structure was solvated in a solvation box using SPC/E water model with $10 \AA$ distance between the edges of the box and the protein fragment. The system was neutralized by replacing water molecules with $\mathrm{Cl}^{-}$and $\mathrm{Na}^{+}$ions. The system was initially relaxed using steepest descent algorithm followed by conjugate gradient algorithm. After energy minimization, the entire system was equilibrated for 100 ps using canonical (NVT) and the isothermal-isobaric (NPT) ensembles. Finally, the equilibrated system 
was simulated for a period of 20 ns using NPT ensemble to understand the effects of aforementioned substitutions on the structural dynamics of Tau. In parallel, the Tau protein was docked to the heparin using ClusPro server to identify its main heparin-binding interface [30]. The docked Tau-Heparin complex was also subjected to the GROMACS program to run a 20 ns MD simulation using aforementioned parameters. A global search for heparin-binding sites on Tau was conducted using the program AutoDock (version 4.2). An ensemble of initial structures for the ligand probe was prepared from [31]. The lowest energy structure from the largest cluster was considered as the best docking solution. The figures were composed using VMD [32]. Electrostatic potentials for Tau were computed using APBS Plugin in Pymol. The schematic diagrams for the interactions between the docked hexa-saccharide and the protein were generated with LIGPLOT.

\section{Results and discussion}

The human central nervous system expresses six low-molecular-weight Tau isoforms that range in size from 352 to 441 amino acids. Human adult Tau has approximately equal representation of 3R and 4R Tau isoforms, with the 1N3R and 1N4R being the most abundant forms [33]. We selected 1N4R isoform because investigation of full-length adult Tau lead to a better understanding of the nature of Tau fibril formation mechanism. Schematic representation diagram of 2N4R and 1N4R Tau isoforms are shown in Figures 1 and 2.

\section{Fig. 2}

Analysis each of nucleation (lag phase reflects the time required for seed formation) and elongation steps [35-37] involved in the Tau fibril formation may be useful for gaining a deeper insight into possible mechanisms of amyloid fibrillation and would be valuable therapeutic targets for the treatment of related diseases. As stated earlier, although heparin is not a perfect model for HS, both heparin and HS induce formation of Tau filaments with very similar morphologies [38]. Also, since the exact mechanism of the inducer in triggering the aggregation of Tau is not fully understood, heparin and its shorter homologue, enoxaparin, were used to investigate whether Tau aggregation under the effect of the inducer length occurs through a 
similar nucleation-dependent polymerization mechanism or not. Enoxaparin is obtained by alkaline depolymerization of heparin benzyl ester derived from porcine intestinal mucosa [39].

\subsection{Amyloid fibril formation by 1N4R Tau in the presence of Heparin and Enoxaparin}

Tau, alone, clearly shows a very high critical concentration for fibril formation, both in vitro [40] and in vivo [41]. Heparin-induced polymerization of 1N4R Tau was analyzed by ThT-binding assay, as most commonly used to assess amyloid assembly in real time. ThT shows enhanced fluorescence at $482 \mathrm{~nm}$ when bound to amyloid fibrils. Tau prepared with inducer induction, generally, displayed an increase in fluorescence signal only after 4-5 h of incubation and a plateau after $\sim 48$ h. [42]. As shown in the Fig. 3, the duration of lag time was relatively large and reciprocally dependent on [inducer] $]_{0}$, at lower concentrations of the inducer. Also, as indicated in Fig. 3A, the fluorescence intensity of ThT increased significantly with increase of heparin concentration (from 9.5 to $16 \mu \mathrm{M}$ ). The same observations were made for enoxaparin, but at different (higher) concentrations. Fig. 3B, also, demonstrates a gradual development of ThT fluorescence signal at $482 \mathrm{~nm}$ with increase in "enoxaparin" inducer concentration, in the indicated concentration range (from 39 to $63 \mu \mathrm{M}$ ).

\section{Fig. 3}

As depicted in Fig. 3, the amyloid formation process was found to obey the characteristic nucleation-elongation pattern, with three distinct phases: initial nucleation, elongation and equilibration, in full agreement with observed aggregation behavior of full-length Tau (hTau40, see Fig. 1) [6] where its assembly were approximated by a simple nucleation-dependent mechanism, and also, reciprocally, inconsistent with reported kinetic aggregation of four repeat domain of Tau (Tau4RD), a truncated Tau isoform, where its aggregation appears to follow single-exponential kinetics with no lag phase [6].

Interestingly, as indicated in the Fig. 3 (and Fig. 4), the aggregation extents then decreased with further increase in concentrations of the both inducers. Also, as expected, Tau prepared with no induction displayed no measurable ThT fluorescence signal both at $t=0$ and $t=96 \mathrm{~h}$, even when 
the protein concentration is $80 \mu \mathrm{M}$, confirming that Tau cannot assemble spontaneously to form amyloid assemblies.

\subsection{Dependency of Tau fibrillation on the concentration/length of the polyanionic inducer}

Heparin as well as enoxaparin promote the aggregation of Tau in a concentration-dependent manner. Various concentrations of the inducers were used for comparing the effects of the inducer concentration/length on Tau protein aggregation. As indicated in Fig. 4 (top section), (as well as Fig. 3), fibrillation (aggregation) extent, the amplitude of the change in ThT fluorescence, of $1 \mathrm{~N} 4 \mathrm{R}$ Tau isoform is sensitive to both the concentration and length of the polyanionic inducer, present in the buffer. Heparin, at most suitable concentration $(\sim 20 \mu \mathrm{M})$, induces much higher aggregation extent, based on ThT fluorescence signal, compared to that of enoxaparin at $\sim 80 \mu \mathrm{M}$ (compare ThT intensities $\sim 600$ for heparin vs. $\sim 220$ for enoxaparin). Binding of heparin (with different MWs) to Tau has been reported to be tight with a dissociation constant less than $1 \mu \mathrm{M}$ $[21,43]$. Regarding the dependency of the extent of fibril formation on the inducer concentration, since at the most effective "protein/inducer" amounts, the ThT fluorescence signals reach limiting (max) values, it may be used to estimate the stoichiometries of protein to the inducers in the rate-limiting steps of aggregation; one heparin molecules appears to bind $\sim 3$ protein molecules while more than 1 enoxaparin molecules interact with each Tau protein. So, regarding to the estimated stoichiometries, we set out to check whether the mechanism of aggregation, induced by heparin and short heparin fragment; enoxaparin, are the same or not. First, there is this possibility that at the most effective "protein/inducer" ratios (at $\sim 20 \mu \mathrm{M}$ heparin/ $\sim 80 \mu \mathrm{M}$ enoxaparin, see Fig. 3 and 4, top and middle sections), there is enough inducer to allow Tau to bind and nucleate to a significant extent before the second inducer binding step occurs; but at higher inducer concentrations, binding of additional heparin/enoxaparin to the growing "Tau/inducer" complexes occurs so rapidly as to block further incorporation of Tau. The same observations were reported for the brain-specific protein; p25 $\alpha$ [44]. As indicated in Fig. 4 (top section), the bell-shaped dependence of the 1N4R Tau fibrillation extent on both heparin and protein concentration are coincident when the ThT fluorescence signals are plotted against the molar ratio of protein to heparin, and, in agreement with earlier reports[6] and [45], the peak of this bell-shaped pattern lies at a ratio of $\sim 3$ or $\sim 4$. However, maximum Tau aggregation was 
achieved at about "Tau/enoxaparin" ratio of 0.7 , indicating that higher concentrations of enoxaparin are needed to induce Tau aggregation. In other words, for enoxaparin, $\sim 1: 1$ protein/enoxaparin ratio is the most effective stoichiometry.

\section{Fig. 4}

Interestingly, there is disagreement between research groups on the effective "inducer/Tau" stoichiometry, triggering protein fibrillization, because of heterogeneity of the inducers (with average MWs of 3000-16000 Da) and Tau isoforms used [21,43,45]. An inconsistent study, also, showed that the small heparin fragment (MW: $4.2 \mathrm{kDa}$ ) was not able to induce Tau filament formation, while still interacting with Tau [21].

The same observations were also made for dependency of apparent aggregation rate on the inducer concentration. Both heparin and enoxaparin accelerated the apparent rate of fibril formation at lower concentrations. But, in agreement with [6], above $\sim 20 \mu \mathrm{M}$ heparin, the apparent rate decreased with further increase in heparin concentration, so that the apparent rate of Tau aggregation also displays a bell-shaped dependence on heparin concentration [6] (Fig. 4, middle section). The same results were also observed for enoxaparin, but above $\sim 80 \mu \mathrm{M}$ enoxaparin the Tau aggregation rate decreased with increase of amount of the inducer. Additionally, by comparing ascending parts and peaks of the curves (see $k_{a p p}$ values in tables 1 and 2), it is evident that aggregation events in the presence of heparin are kinetically faster than those for enoxaparin. Additionally, in accordance with [6], when the molar ratios were kept fixed and the protein and inducers concentrations are varied accordingly, the aggregation rate remains the same, proving that the role of stoichiometry is not incidental but rather crucial to the aggregation process (data not shown).

\subsection{Tau oligomer nucleation under the effect of the inducer length}

The sigmoidal shapes of amyloid aggregation (see Fig. 3) showing well-defined lag period followed by a sharp increase in ThT fluorescence and/or turbidity involve nucleation, where unstable and soluble pre-fibrillar intermediates precede the formation of stable amyloid fibrils [46]. Heparin appears to support heterogeneous Tau nucleation so that it facilitates filament nucleation on its surface [18]. Also, the anionic inducer is suggested to promote conformational 
change leading to the formation of nucleation-competent oligomeric subunits [6]. In the classic equilibrium nucleation-elongation model, assembly-competent monomer is in rapid equilibrium with a thermodynamically unstable species (nucleus). There is the possibility that "nucleation" changes under the effect of the inducer length. As shown in Fig. 4 (bottom section), the duration of lag time was relatively large and, at least at lower concentrations, reciprocally dependent on the heparin concentration, but above $\sim 20 \mu \mathrm{M}$, the lag period increased with further increase in heparin concentration, so that the nucleation time of Tau aggregation displays a V-shaped dependence on heparin concentration [6]. Although much higher concentrations of the LMW inducer, enoxaparin, at the most appropriate stoichiometry, is needed for the amyloid nucleation, both inducers appeared to be equivalent to induce Tau nucleation, as confirmed by descending parts of the Fig. 4 (bottom section) and Lag time values, listed in tables 1 and 2. Additionally, differential increase of lag periods at the ascending parts of the V-shaped curves (see Fig. 4 (bottom section) also tables 1 and 2) was also documented which indicates that even interfering behavior of the inducers is under the effect of those MWs, as well. So, as stated earlier, it appears that binding of additional enoxaparin to the growing "Tau/inducer" complexes occurs so faster (compared to heparin) to block further incorporation of Tau. Furthermore, we can also confirmed that the inducers are acting as allosteric modulators (see [23]), both as activators at low concentrations and inhibitors at high concentrations.

However, as stated in the experimental section in details, plots of blank-subtracted ThT fluorescence values versus time were analyzed with a procedure of best fit, using an appropriate single sigmoidal function and the values of lag time, apparent aggregation constants and extent of aggregation, for Tau in the presence of heparin and enoxaparin, were obtained from the related sigmoidal plots and are listed in tables 1 and 2. As indicated quantitatively, an increase in the inducers concentration resulted in a decrease in the lag time and an increase in the apparent rate constants and fibril extent but, in agreement with $[\underline{23}, \underline{44}, 6]$, above the critical concentration, the results were reversed (the lag time increased while the apparent rate/aggregation extent decreased.

\section{Table 1}

Table 2 


\subsection{Does aggregation mechanism change under the effect of the polyanionic inducer}

length?

Additional comparative characterization of kinetic effects of heparin and enoxaparin on Tau amyloid aggregation was performed by plotting scaled ThT fluorescence against scaled time. Analysis of aggregation of Tau protein in the presence of heparin/enoxaparin was accomplished in the range of total inducer concentrations from 10-44 and 40-174 $\mu \mathrm{M}$, respectively. We then analyzed the kinetic data by plotting scaled ThT fluorescence (each ThT fluorescence value divided by the final ThT fluorescence intensity at specific inducer concentration $\left(F / F_{\max }\right)$ ) against scaled time (each time divided by the time needed to reach half-amounts of aggregates or half of the limiting ThT fluorescence at corresponding inducer concentration $\left(t / t_{D}\right)$ ) (Fig. 5). As indicated by the figure, since the different series fall on a single curve, it can be deduced that the underlying aggregation mechanism is relatively simple (at different concentrations of inducers) and the differences amongst the series are related to different time and fluorescence scales $[46,47]$. Also, comparing scaled data of heparin with those of enoxaparin, it appears that the mechanism of aggregation reaction remains un-affected upon change of the inducer length (MW).

\section{Fig. 5}

Superposition of the kinetic curves (Scaled Data) means the existence of correlation between $k_{\text {app }}$ and $t_{\mathrm{D}}$. Thus, similar to the " $F / F_{\max }$ versus $t / t_{\mathrm{D}}$ " plots, the " $k_{\text {app }}$ versus $t_{\mathrm{D}}$ (or $1 / t_{\mathrm{D}}$ )" plots can be used. Correlation between the aggregate growth rate $\left(k_{\mathrm{app}}\right)$ and the lag period $t_{\text {lag }}$ was previously discussed by Fändrich [48]. According to this study, Fig. 5C which is based on the tabulated data, suggests that heparin-induced aggregated samples, at all concentrations, possess kinetic properties similar to those samples induced by enoxaparin. The applicability of this equation for amorphous aggregation was also recently discussed by Kurganov's research group [49].

\subsection{Fibril formation behavior, for both inducers, is also reproducible under the effect of Tau concentration}

Previous data (dependence of the aggregation kinetics on the inducer concentration) may lead us to conclude that adoption of assembly-competent conformations, associated with enriched $\beta$ sheet content, can be driven by both heparin and enoxaparin through a similar mechanism that 
approximates heterogeneous nucleation. So that the extent of Tau aggregation strictly depends on Tau/inducer ratios. The observation made in this study that both the extent and apparent rate constant for fibril formation increase with the inducer concentrations and reach their maxima values when the protein:inducer ratios are 3:1 and $\sim 1: 1$ for heparin and enoxaparin, respectively. Since the stoichiometries between two partners (Tau protein and HMW/LMW inducers) were so different and influenced fibrillation dramatically, these observations could explain, in part, the mechanistic basis for this dependence.

To examine the effect of protein concentration, fibril formation of Tau protein (measured by the following the temporal development of ThT fluorescence) in the range of total protein concentrations from 16 to $95 \mu \mathrm{M}$ was studied in the presence of $16 \mu \mathrm{M}$ heparin (Fig. 6A) and 63 $\mu \mathrm{M}$ enoxaparin (Fig. 6B). The ThT fluorescence monitored aggregation kinetics was found, again, to obey the characteristic nucleation-elongation pattern, at all protein concentrations, with three distinct phases: initial nucleation, elongation and equilibration. Additionally, only at lower Tau concentrations, the duration of lag time was relatively large and reciprocally dependent on $[\mathrm{P}]_{0}$. Both apparent aggregation rate constants and aggregation extents show a bell-shaped dependence on protein concentration (Figs. 7 and S1) with peaks at the protein concentrations of 50-60 $\mu \mathrm{M}$, for both the inducers. Furthermore, for better quantitative assessment of data, plots of blank-subtracted ThT fluorescence values versus time were then analyzed with a procedure of best fit, using an appropriate single sigmoidal function and the values of lag time, apparent aggregation constants and extent of aggregation, for Tau in the presence of heparin and enoxaparin, were obtained from the related sigmoidal plots and are listed in tables 3 and 4 . As indicated in the Fig. 6 (and tables 3 and 4), the aggregation rates/extents then decreased with further increase in the protein concentration.

Fig. 6

Fig. 7

Table 3

Table 4 
To determine apparent aggregation reaction order with respect to Tau protein, the initial velocity of ThT fluorescence production was measured as a function of Tau concentration. Initial slopes of aggregation were then calculated by analyzing the terminal phase of the kinetic curves $[46,50]$, and the initial part of each curve, usually including a lag period, was ignored, so the rate of appearance of ThT fluorescence was assumed to reflect the formation of prefibrillar (such as protomer) species. Replots of these data in double log format revealed a slope of $\sim 1$, indicating that the appearance of ThT fluorescence is first order with respect to Tau protein concentration (Fig. 7). The first-order kinetics of Tau aggregation is consistent with this idea in which the ratelimiting step of aggregation process is the intra-molecular conformational changes of each independent polypeptide and, in other words, suggest that the intermediate corresponds to a conformation of monomers. The same observations were also made for enoxaparin and Tau aggregation order induced by the heparin fragment was calculated close to 1 (data not shown). The similar (calculated) values of aggregation order parameters " $n$ ", also can confirm that ratelimiting step (RLS) of the aggregation mechanism is not seriously affected under the effect of the inducer length. These observations are in accordance with the results reported by Chirita et al. [17].

Again, here, the aggregation rate constants are plotted against the molar ratio of protein to the inducer. In agreement with Fig. 4 (middle section) data, the bell-shaped dependence of the fibrillation rate constants was observed and the peaks of these bell-shaped patterns were registered at a ratio of $\sim 3$ for heparin and $\sim 0.7$ for enoxaparin. Additionally, when the molar ratio is kept fixed and the protein and heparin concentrations are varied accordingly, the rate remains the same, proving that the role of stoichiometry is not incidental but rather crucial to the aggregation process. Moreover, the longer chain of the inducer, the larger is the protein/inducer ratio (Fig. S1).

Additional comparative characterization of kinetic effects of the protein concentration on Tau amyloid aggregation, in the presence of $16 \mu \mathrm{M}$ heparin and $63 \mu \mathrm{M}$ enoxaparin, was performed by plotting scaled ThT fluorescence against scaled time, in the range of Tau concentrations from 16$95 \mu \mathrm{M}$. We again analyzed the kinetic data by plotting scaled ThT fluorescence against scaled time (Fig. S2). As indicated by the figure, since the different series fall on a single curve, it can be deduced that the underlying aggregation mechanism is relatively simple (at different 
concentrations of the protein) and the differences amongst the series are related to different time and fluorescence scales. Also, slight differences between scaling properties of two independent series, in the presence of heparin and enoxaparin, indicate that mechanism of aggregation reaction appears to be un-affected under the effect of the inducer length.

\subsection{Heparin-induced $\beta$-structured intermediates are more hydrophobic}

Because the apolar residues generally make up 30-50\% of most proteins, a considerable amounts of protein (stability and) folding's driving force must be derived from hydrophobic interactions [51]. Moreover, with regard to kinetic competition between correct folding and undesired aggregation [52]; it appears reasonable that hydrophobic effects should be also involved in unproductive protein misfolding and aggregation. In that respect, several authors [53] have considered a key role for the hydrophobic interactions in the early stages of amyloid formation. Need to further insight in this regard, led us to evaluate the potential involved interactions, at the early stages of polyaninon-induced Tau fibrillogenesis. When ANS binds to solvent-exposed hydrophobic clusters, it generates a remarkable increase in its fluorescence intensity and a blue shift of its maximum emission wavelength [53,54]. As it is evident from Fig. 8, and in agreement with [55], ANS fluorescence intensity gradually increased with time, when Tau was incubated with the inducers and, in accordance with ThT fluorescence data, reached a steady-state level at $\sim 48 \mathrm{~h}$. The enhanced ANS fluorescence intensities suggest that the related fluorophores become more exposed to the solvent while native Tau molecules are being converted to aggregation-competent species. Such enhanced emission intensity is also attributed to decrease in the internal relaxation of the ANS fluorophores [53]. Furthermore, as it is evident from the figure, after heparin-Tau interaction, more assembly-competent (amyloid protofibril) species with higher exposed hydrophobic areas, compared to those of enoxaparin, are generated. Such conditions may encourage intermolecular hydrophobic interactions among the early heparin-induced intermediate structures and, in agreement with ThT registered kinetics (Fig. 3, Fig. 6), trigger Tau fibrillation for prepared samples induced by the longer inducer. However, here, fluorescence intensity of both dyes (ThT and ANS, detecting $\beta$-sheet content and exposed hydrophobic patches, respectively) did not increase with time for Tau incubated without heparin, 
confirming failure of Tau alone to spontaneously aggregate over the experimental time period is documented.

\section{Fig. 8}

\subsection{Are the inducers transient or permanent partners of Tau?}

We clearly showed that Tau-binding behavior (stoichiometry) of polyanionic inducers are different at initial stages of the protein amyloid aggregation. We also show here that heparin accelerates the assembly of Tau protein into fibrils, although there is still debate whether it remains associated with the fibrils produced, as permanent template, or not, but all previously published data are in full agreement (with each other) that the inducer acts as a template and/or nucleation catalyst that facilitates assembly of Tau into fibrils. Using fluorescently labeled heparin, some reports indicated that heparin serves only as sources of nucleation is not incorporated within the core of the filaments [24] and [23]. On the other hand, other papers showed that heparin becomes integrated into the rigid core region of the PHF, probably providing the charge compensation for the lysine-rich stretches that form upon the in-register, parallel stacking of the repeat regions [21] and [43].

The dissociation constant for Tau bound to heparin is less than $1 \mu \mathrm{M}$ [21] and, apart from the polyanion length, it appears that this value is not so different for enoxaparin. Hence, when the binding of protein to the inducer, as monitored by the amplitude of ThT fluorescence change accompanying the aggregation reaction, is carried out at protein concentrations greatly exceeding the dissociation constant, the binding curve yields not the dissociation constant but only the stoichiometry of protein bound to the inducer [56]. As stated earlier, the amplitude of the change in the ThT signal has a linear dependence on the protein amount (at lower concentrations) where the apparent rate constant increases, but at very high protein concentrations the ThT fluorescence signal does reach a limiting value. Also, we supposed that protein (P) in protein-heparin (PH) binary system as well as in PHP ternary system is as capable of binding ThT as is $\mathrm{P}$ in the fibrils at final stage [57]. As indicated in Fig. S3, only 1 heparin molecule is bound to 15 protein molecules when fibril formation is complete while Tau/enoxaparin stoichiometry at final stage reaches 1:5 (one enoxaparin molecule per 5 Tau molecules). This may inspire us to conclude that a; some inducer molecules are liberated out 
during amyloid aggregation (e.g. initial 1:3 heparin/Tau ratio vs. final 1:15 ratio), b; final states of the protein in the presence of the inducers may be structurally different, possibly in agreement with non-identical amplitudes of ThT fluorescence changes (see Fig. 3). This issue will be more clarified by AFM imaging method.

\subsection{Does critical concentration for aggregation depend on the inducer length?}

As the fibrillization reaction reaches equilibrium, the rate of protein addition equals the rate of protomer dissociation from filament ends. This final equilibrium is characterized by a "critical" concentration corresponding to the maximal solubility of protein above which all additional protein protomers enter to polymeric phase, and is another characteristic of nucleationdependent reactions. This parameter which is the highest concentration of protein that does not support amyloid aggregation, and therefore is frequently corresponds to the abscissa intercept of the Tau concentration dependence curve, can be calculated from any assay modality, such as light scattering, ThT/ThS fluorescence and electron microscopy (EM), although EM methods ensure that the measurements are specific for filaments rather than other species [58].

Some differences between heparin and enoxaparin (such as unequal extended negative charges) may affect interaction of these polyanions with Tau, leading to diverse conformational switches and protomers. We therefore set out to compare critical protein concentration in the presence of heparin with that of enoxaparin. To determine the relationship between " $\beta$-structured intermediate" formation and filament critical concentration, Tau fibrillization (based on ThT fluorescence intensity at equilibrium), was found to be directly proportional to Tau monomer concentration, in the presence of both heparin and enoxaparin (Fig. S4). Linear regression analyses of the "aggregation extent dependence on Tau concentration" data provides a value of $\sim 3.3$ (abscissa intercept for heparin) and $\sim 2.6$ (abscissa intercept for enoxaparin) $\mu \mathrm{M}$ for the critical concentration; similar regression analysis of the apparent rate constant dependence on Tau concentration provides the similar observations (data not shown), indicating that "critical concentration" undergoes no significant change under the effect of the inducer length. This value is consistent with previous estimates and indicates that the final equilibrium between filament ends and unincorporated Tau is similar in the presence of diverse anionic inducers. The abscissa intersection by curve at $\sim 3 \mu \mathrm{M}$, also, may indicate that formation of $\beta$-sheet enriched assemblycompetent intermediate needs a critical concentration of Tau protein. These data are in 
agreement with the ThT-reactive intermediate being at least a dimer that requires a minimal concentration for formation [17]. In confirmation of a critical concentration below which Tau does not aggregate, Ramachandran and Udgaonkar also found that at protein concentrations of 2 and $3 \mu \mathrm{M}$, no increase in ThT fluorescence was seen even at $100 \mathrm{~h}$, indicating that aggregation does not occur at these protein concentrations [6].

Tau aggregation was allowed to proceed for up to $96 \mathrm{~h}$. Aggregation of Tau over time in the presence and absence of inducers was comparatively monitored by SDS-PAGE, CD, XRD spectroscopy and, atomic force microscopy (AFM), then each sample/fraction was characterized with respect to $\beta$-sheet content, and fibril appearance/dimension.

\subsection{Monitoring Tau aggregation (intermediates) by SDS-PAGE}

To obtain more precise information on the size distribution and kinetics in the formation of Tau aggregation intermediates, aggregates were sampled after different periods of time and subjected to SDS-PAGE analyses. On SDS-PAGE, monomeric Tau runs as a single band at apparent MW around $60 \mathrm{kDa}$. At various time intervals of Tau incubation with the inducers, additional bands occurred in the range of $\sim 150-200 \mathrm{kDa}$ and (band numbers as well as intensities) increased, relatively, with further incubation time (Fig. 9). However, higher MW Tau filaments did not enter the stacking gel and therefore could not be quantified via SDS-PAGE. In agreement with the previously reported results [55], the densitometric analysis of Tau bands (and smears) above the monomer band, representing Tau oligomer species, affirms the increase of Tau aggregation in the presence of heparin with increasing incubation time. We also found that various kinds of Tau oligomers (including dimers/trimers) are significantly populated, in the presence of heparin, while enoxaparin failed to induce SDS-stable aggregation products, confirming that heparin (not enoxaparin) is capable to form more oligomer species with a metastable supramolecular packing, possibly with enriched $\beta$-sheet content (detectable by ThT-binding assay), before final fibrillization. The SDS-PAGE technique also confirmed aggregated Tau above the monomer band

occurs only marginally with proceeding incubation time, in the absence of heparin, confirming that the innate ability of Tau to aggregate in vitro into high molecular weight species is low.

\section{Fig. 9}


Quantification of the protein monomer bands using ImageJ, a scientific image analysis software, and approximate intensities of DNA band areas are also shown. As indicated in Figs. 9 and S5, intensity/appearance of the Tau monomer bands reduced with increasing incubation time due to the formation of aggregated, high molecular weight Tau species. Interestingly, Quantification data showed that Tau monomer content, in the presence of enoxaparin, decreased more than that of heparin, as a function of aggregation time (Fig. S5)

As stated earlier, heparin plays a role in the activation of Tau molecules leading to the formation of nucleation-competent oligomeric subunits (or amyloid protofibril nucleuses). Some researchers [18] believe that amyloid protofibril nucleus may be a dimeric species since certain rare Tauopathies accumulate primarily three-repeat Tau isoforms, whereas most others accumulate four-repeat isoforms. The dimer may span the core of the Tau filament, which adopts parallel, in register $\beta$-sheet conformation and which is composed of 2 Tau protomers per $\beta$ strand spacing [18]. The SDS-PAGE analyses also indicated the presence of SDS-stable Tau dimers/trimers, especially when heparin was used as the inducer, and reciprocally these types of $\beta$-sheet enriched assembly-competent intermediates (Tau oligomers) was not populated in the presence of enoxaparin. On the other hand, as indicated in Fig. 3 and tables 1 and 2 (note the lag time values), both heparin and enoxaparin were successful to facilitate filament nucleation on those surfaces. But we observed significant acceleration of Tau fibrillization by heparin and low aggregation tendency of the protein in the presence of enoxaparin, based on ThT fluorescence signal, while heparin caused a small increase in filament nucleation rate, compared to enoxaparin. There is the possibility that, in case of the shorter inducer; enoxaparin, side reactions, such as the formation of species off the aggregation pathway, populates $\beta$-sheet deficient aggregation-incompetent intermediates. As evidenced by SDS-PAGE technique (Fig. 9 and Fig. S5), and regarding higher enoxaparin concentration used, these diverse SDS-insoluble oligomeric assemblies are more populated and they would be expected to contribute to monomer depletion and, therefore, to change aggregation kinetics and filament length distributions. In agreement with this possibility, smaller heparin fragments $(4.2 \mathrm{kDa})$ have been found to be unable in inducing on-pathway aggregation of the full-length Tau while still interacting with Tau [21]. 


\subsection{Far-UV CD Measurements}

We used Far-UV CD spectroscopy to gain insight into secondary structural characteristics of amyloid aggregates, under the effect of inducer length. At the start of the aggregation reaction, the soluble protein exhibited a predominantly monomeric random coil structure, a minimum around $200 \mathrm{~nm}[45,60]$. After $96 \mathrm{~h}$ incubation of Tau protein with the inducers, and in order to detect the actual difference between the spectra, amyloid aggregates were isolated from the residual soluble protein in the reaction by centrifugation prior to CD spectroscopy. A transition from random coil to a beta-sheet structure was detected in the presence of heparin and enoxaparin (Fig. 10). The spectra of the protein aggregate samples, in the presence of both inducers, exhibit clear shift of the minimum to higher wavelengths, a broad minimum at 215 $\mathrm{nm}$ as typical feature of $\beta$-sheet structure, whereas in the absence of inducers, Tau dose not exhibit any secondary structural change (Tau prepared without heparin induction displayed no aggregation). To come up with a correlation between the structural level and the extent of amyloid aggregation (see earlier sections), we compared the secondary structural variation of the protein under the effect of the inducer length. As it is evident in the figure, heparin caused much greater negative ellipticity in the aggregated Tau complex, compared to that of enoxaparin, confirming that heparin-Tau interaction leads to more successful fibril formation with higher $\beta$ sheet content compared to that of enoxaparin.

\section{Fig. 10}

\subsection{FTIR analyses}

The CD result was confirmed by the analysis of the soluble and polymerized protein by FTIR spectroscopy. This technique is less sensitive than CD to scattering effects induced by aggregation, and has been widely used to assess the secondary structure of proteins [61]. The soluble protein exhibits a maximum at $1650 \mathrm{~cm}^{-1}$, consistent with random coil structure, whereas the aggregate samples peaks at $1632 \mathrm{~cm}^{-1}$, also clearly indicating an increase of beta structure (Fig. 10) [6]. Hence, the data suggest that Tau fibrils induced by heparin and enoxaparin have similar $\beta$-sheet structures, but with different extent. 


\subsection{XRD analyses}

The most reliable demonstration of fibril-like structure is provided by XRD data, which directly report on the molecular structure and presence of amyloid cross- $\beta$ structure, so that is a powerful technique, used for determining the nature of protein aggregation. Classical fibrils show typical diffraction patterns at around 4.5-5 (corresponding to inter-strand distances) and 9.5$11 \AA$ (inter-sheet distances) [62-66]. D-space of $\sim 4.7 \AA$, (the distance between hydrogen-bonded $\beta$-strands) and especially d-space of $\sim 10 \AA$ that arises from association of the sheets and is dependent on the size of side chains [66-70], are not fixed values and vary for different amyloidogenic proteins as well as with changes in fibrillation conditions. Results of XRD analysis of heparin- and enoxaparin-treated Tau samples are shown in Fig. $11 \mathrm{~A}$ and B, respectively, as plots of scattering intensities versus the scattering angle $2 \theta$. XRD pattern of heparin-treated Tau sample exhibited two peaks, corresponding to d-spacings of $4.7 \AA$ (denoted by peak of $2 \theta$ around $20^{\circ}$ ) and $10.4 \AA$ (denoted by peak of $2 \theta$ around $10^{\circ}$ ), indicating the respective inter-strand and inter-sheet distances in cross- $\beta$ structure [62,71]. Calculations for $d$-spacings were carried out using Bragg's law of diffraction [63]. The organization of the $\beta$-structure within the enoxaparintreated Tau was also analyzed by X-ray diffraction. As shown in Fig. 11B, enoxaparin-treated Tau sample displayed clearly discernible pattern of scattering intensity peaks of $2 \theta$ around $20^{\circ}$ as well as and $10^{\circ}$, characteristics of cross- $\beta$ sheet structure, but with much lower intensity. These results further confirm the amyloid nature of aggregation in Tau and strengthen the findings obtained by other experimental (ThT, CD and FTIR) methods, confirming that the longer inducer strongly trigger Tau amyloid aggregation. Additionally, the fact that almost one strong intersheet peak is present could indicate that only one fibril morphology is present in solution, especially for heparin-treated Tau sample. These peaks were also absent in the XRD pattern of control Tau, indicating Tau without inducer completely failed to form amyloid aggregate. (Fig. 11B, inset). The obtained results are in full agreement with earlier report [45] in which pronounced reflections centered at $4.7 \AA$ on the meridian (the distance between two adjacent strands in a $\beta$-sheet) and $10.7 \AA$ on the equator (which typically stems from the inter-sheet stacking of two $\beta$-sheets) were observed.

\section{Fig. 11}




\subsection{Characterization of fibril morphology}

As observed before [38], incubation of the Tau isoform with heparin led to bulk assembly into twisted filaments with a morphology similar to the PHFs from Alzheimer's disease brain. The fibril formation in the presence of heparin and enoxaparin were verified using AFM. In the absence of inducers no aggregate was observed, while incubation of Tau in the presence of heparin and enoxaparin for $120 \mathrm{~h}$ at $37{ }^{\circ} \mathrm{C}$ resulted in the protein assembly (Fig. 11, bottom). Incubation of Tau with heparin gave large numbers of twisted filaments, while enoxaparin induced the formation of $\beta$-structured (based on ThT studies) short fibrillar assemblies, with appearance similar to amorphous oligomeric aggregates. In full agreement with XRD pattern, the results of AFM images show that heparin and enoxaparin induced formation of Tau assemblies with different extent of extended $\beta$-structures.

\subsection{Computational studies}

Based on our results and the published data [21,43], it is suggested that heparin binds to fulllength human Tau mainly through its segment 244-372, triggering Tau fibrillization. Root mean square deviation (RMSD) is most often used for evaluating the flexibility differences between protein structures and considers as an indicator of structural stability. During the whole simulation time, the RMSD value of Tau alone was relatively higher than that of "Tau-heparin" with the average value of $\sim 0.90 \mathrm{~nm}$ and $0.55 \mathrm{~nm}$, respectively. So RMSD plots of the Tau and Tauheparin complex revealed that the stability of the protein monomer is considerably increased when binds to the heparin (Figure S6). This state can be defined as the least stable species reversibly interconverted with assembly competent monomer. In this regard, it is already suggested that enoxaparin and heparin are capable to reduce thermodynamic barrier by binding to Tau and induce/stabilize assembly-competent conformations [43].

Molecular docking was applied to determine the heparin-binding region of Tau. There were two distinct heparin-binding interface in the Tau. However, the main heparin-binding pocket of Tau is located at the position 327-391 (Fig. 12). This result is in accordance with data, reported by Sibille et al. [21]. They discovered this segment of Tau sequence, as another site of heparin 
interaction, in the region following the fourth repeat (R4, residues 336-370) and a remarkable cluster in the proline-rich region just preceding the MTBR.

\section{Fig. 12}

They suggested that heparin becomes integrated into the rigid core region of the PHF, probably providing the charge compensation for the lysine-rich stretches that form upon the in-register, parallel stacking of the repeat regions [21]. Sibille et al. were able to rank the strength of interaction for several other residues in the full-length Tau. The ( $\left.\mathrm{V}_{306} \mathrm{QIVYK}_{311}\right)$ within R3 and specifically Lys ${ }^{311}$ as well as Lys ${ }^{257}$ and Ala ${ }^{152}$ were reported as the most important binding sites for the short heparin fragment. They also suggested that the fragment $\left(\mathrm{V}_{275} \mathrm{QIINK}_{280}\right)$ likewise binds strongly to heparin, specifically Lys ${ }^{347}$, Lys ${ }^{369}$ and Lys ${ }^{395}$ are the other sites of strong interaction. Another study [72] has shown that heparin binds to the hexapeptide motifs in the repeat domain, as well as to positively charged patches such as the KK motif at the beginning of R2 $\left(280 \mathrm{KK}_{281}\right)$ and the $\mathrm{KH}\left(267 \mathrm{KH}_{268}\right.$ and $\left.{ }_{298} \mathrm{KH}_{299}\right)$ or HHK $\left.{ }_{329} \mathrm{HHK}_{331}\right)$ motifs near the end of the repeats R1, R2, and R3, so that the binding to heparin is of electrostatic nature; positively charged residues are present as follows: $\operatorname{Arg} 211, \operatorname{Arg} 242, \operatorname{Lys}^{280}, \operatorname{Lys}^{281}, \operatorname{Lys}^{298}, \operatorname{His}^{299}, \operatorname{Lys}^{311}, \operatorname{His}^{329}, \operatorname{His}^{330}$,

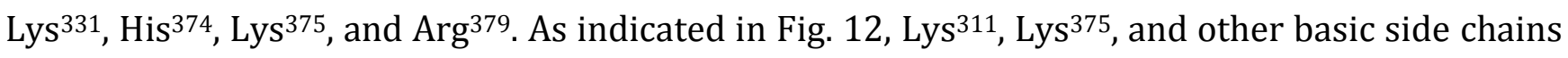
(within 327-391 segment) surround heparin molecule.

The structural dynamics of the native and mutant Tau structures were studied to elucidate the functional diversity of Tau. To that end we measured the root mean square fluctuation (RMSF) of carbon alpha of Tau protein between the trajectories generated at $300 \mathrm{~K}$ to gain insight into the flexible nature of the native Tau protein. The RMSF simulations were plotted and are shown in Figure 12. The obtained RMSF graphs indicated that the flexibility, especially at the main heparinbinding site, of Tau protein is decreased during MD simulations after binding to the inducer. Calculation of solvent accessibility surface area (SASA) also demonstrated that the surface accessibility of Tau protein is reduced after binding to the heparin (Fig. S7). This suggests that the binding of heparin to Tau causes a considerable reduction of Tau interaction with its surrounding solvent molecules. 
It is now widely accepted that a reduced protein surface accessibility can increase the protein stability. Indeed, the reduced protein surface accessibility increases the rigidity and compactness of the protein resulting in less interaction with other macromolecules [73]. The backbone and side chain solvent-accessible areas were also calculated using GETAREA 1.1 [74]. Although, the total surface accessibility of Tau was decreased through binding to heparin, the surface accessibility of Tau's aggregation prone segments ( $\mathrm{V}_{306} \mathrm{QIVYK}_{311}$ and $\left.\mathrm{V}_{275} \mathrm{QIINK}_{280}\right)$ was increased causing a more successful Tau-Tau dimerization and acceleration of amyloid fibrils (PHF) assembly (Table 5).

\section{Table 5}

Recall that short peptides $\left(\mathrm{V}_{306} \mathrm{QIVYK}_{311}\right)$ and $\left(\mathrm{V}_{275} \mathrm{QIINK} 280\right)$, as minimal Tau-Tau interaction motifs, are truly acting as nucleators for the PHF formation. Indeed, heparin (not enoxaparin) has been shown experimentally to induce conformational changes in Tau, and also a role in regulating the conformation of Tau has been proposed earlier [23]. Additionally, in agreement with previous reports [6](and reference 38 therein), the MD/ASA data of the current study clearly suggest that heparin functions by binding $\left(\mathrm{V}_{306} \mathrm{QIVYK}_{311}\right)$ and $\left(\mathrm{V}_{275} \mathrm{QIINK}_{280}\right)$ sites, to be more exposed, and populating/facilitating $\beta$-strand interactions across different Tau molecules, mainly through reducing electrostatic repulsion between interacting Tau molecules.

\subsection{Proposed model for Tau-inducer interaction}

A proposed model shown as scheme 1, is based on inducer-mediated nucleation-dependent mechanism and according to the kinetic and thermodynamic data and the previously reported results [24,43,54]. In this study, as well as in other previous studies [6] (and references 20,28,29,45,62 therein), both a dependence of aggregation extent (based on ThT/XRD signals) on protein/inducer concentration are observed. Also, although sulfation is important in accelerating amyloidogenesis, we revealed that the extent of acceleration is proportional to the molecular weight of heparin, as previously reported for the heparin-gelsolin interaction [75]. The conformational change within Tau monomers are the slowest steps, but the heparin-bound dimers function as nuclei of on-pathway aggregation process. Additionally, since ThT fluorescence saturates at a heparin concentration approximately half that of the protein present 
(and at Tau/enoxaparin molar ratio of $\sim 1$ ), a complex of one heparin molecule with two boundTau molecules, as PHP ternary system or Tau-Tau dimer, (as well as 1:1 stoichiometry for Tau:enoxaparin) are shown in this proposed model. So this study demonstrates that, in presence of the shorter inducer, enoxaparin, aggregation-competent non-covalent dimer (which has a important kinetic role in Tau amyloid aggregation) is not populated. As reported earlier, ( $\left.\mathrm{V}_{306} \mathrm{QIVYK}_{311}\right)$ and $\left(\mathrm{V}_{275} \mathrm{QIINK}_{280}\right)$ amyloidogenic segments of Tau contain important binding sites for heparin. Thus, we can assume that the "inducer binding site" and "segments responsible for Tau-Tau dimerization (assembly)" are almost the same. Structural and conformational changes of Tau increase the solvent exposure of the aggregation-initiating components of the protein, enhancing the probability for intermolecular contacts between these regions $[21,76]$. Also (as shown in scheme 1), it appears that strength of Tau-Tau association is much higher than Tau-inducer`s binding affinity, so due to strong Tau-Tau interaction, especially at the final stages of amyloid assembly, a fraction of Tau-bound inducer molecules are squeezed-out (see ref. [6] and Fig. S3) and released into bulk surrounding solvent. It is suggested that extension of $\beta$ structure, monomers addition to PHPs (accompanied by PHP-PHP interaction followed by monomer addition) happening simultaneously in the elongation step. Also as revealed in the scheme 1, heparin (and to a certain extent; enoxaparin) has much more opportunity to form the aggregation-competent dimer (Tau protomer), which then acts as an efficient nucleus for further growth. Elongation then occurs by a monomer addition mechanism, wherein individual protein molecules, more likely, add to the growing $\beta$-sheet enriched fibril. Reciprocally, non-productive protein-enoxaparin complexes are populated so that this off-pathway step accounts for the observation that fibril formation is slowed down compared to that of heparin, as documented by Fig. 3.

\section{Scheme 1.}

Hasegawa et al. [38] have found that the degree of sulfation is an important variable that determines the Alzheimer-like interactions of glycosaminoglycans with Tau protein. Here, we highlight importance the other determinant variable; "inducer length". Heparan sulphate (HS) is a linear polysaccharide and in common with other glycosaminoglycans has repeating disaccharide unites (50-150) with three parts in its sequence; non-sulphated region, mixed 
sequence and high-sulphation (S-domain) [77]. Heparan sulphate proteoglycans (HSPGs) as the major component of extracellular matrix (ECM) in mammals, are also well-recognized as regulator of cell adhesion and migration [77]. Taking overall similarities between HS and heparin into account, however, and as shown in Fig. S8, there are remarkable differences in molecular properties between heparin and HSPGs (i.e., such as degree of polymerization, conformation of sugar residues, and charge distribution). The HSPG obtained from human liver tissue is a huge macromolecule with MW of $\geq 200 \mathrm{kDa}$ as estimated by SDS-PAGE [78]. Porcine intestinal HS and human liver HS both showed an average molecular weight of $\sim 20$ and $\sim 24 \mathrm{kDa}$, respectively. So, Higher MW of HS, compared to the heparin, may be considered as a potential triggering factor of Tau assembly, in vivo. HSPG may also facilitate Tau filament nucleation on the various S-domains so that accelerates Tau fibrillation kinetics in AD-affected brain cells. Furthermore, due to heterogeneous nature as well as high MW of HSPGs, the inducer fails to squeezed-out during the protein fibrillation so that Tau tangles usually contain components of the basement membrane.

\section{Conclusion}

It is well established that Tau aggregation is one of main responsible factors in AD pathogenesis. Heparin, as an acceptable representative of heparan sulphate is of considerable interest as a causative agent for Tau aggregation, but, to the best of our knowledge, little is known about the molecular details of heparin-induced aggregation, i.e. exact molecular mechanism of heparininduced Tau fibril formation is not fully understood because there is still debate about whether heparin plays a role only in the nucleation step or is either required in both nucleation and elongation steps of Tau fibril formation in vitro. In this comparative study, we assessed Tau aggregation in the presence of two polyanionic inducers; heparin and enoxaparin and proposed a mechanistic model of nucleation. It appears that HMW heparin is more successful to interact both with peptides in the second and third repeats (R2 and R3) and with the basic regions flanking the MTBR, through structural changes in the former and charge neutralization followed by an overall structural change in the latter, enables efficient aggregation of Tau into mature Tau filament. Reciprocally, enoxaparin has been found to be unable in inducing on-pathway aggregation of Tau while still interacting with Tau, so this off-pathway step accounts for the observation that fibril formation is slowed down compared to that of longer heparin molecule. 
Enoxaparin is a heterogeneous mixture of shorter polysaccharides, produced by depolymerization of the heparin polysaccharides. The main difference heparin and enoxaparin appears to be only those lengths, since the depolymerization process results in selective cleavage between the uronic acid and glucosamine residues, so that sequence of disaccharide building blocks remains unchanged. Furthermore, heparin and enoxaparin may exhibit small differences for $\mathrm{pKa}$ of the acidic functional groups. So we propose that differences in the secondary structure may play a role in the observed differences in $\mathrm{p} K_{\mathrm{a}}$. The heparin may adopt a helical coil, whereas the smaller oligosaccharides are unable to achieve such a secondary structure, so these possible conformational differences can explain, in part, interaction inequalities between the inducers and Tau and different aggregation propensities of the amyloidogenic protein.

\section{Acknowledgments}

The authors gratefully acknowledge the Research Council of Kermanshah University of Medical Sciences. We also would like to thank IRAN National Science Foundation (INSF) for the financial supports (Grant No. 95815088). R.K. kindly acknowledge Dr. Markus Zweckstetter as well as Shaolong Zhu and Dr.Derek J. Wilson for providing the pdb coordinate file for the 'native' Tau ensemble predicted from their NMR/HDX work and are also very grateful to Dr. J. Avila (Universidad Autonoma de Madrid, Spain) for Tau (1N4R) plasmid. Effective, instructive and invaluable comments/assistants, provided by anonymous reviewers are gratefully acknowledged.

\section{References:}

[1] R.N. Rambaran, L.C. Serpell, Amyloid fibrils, Prion 2 (2008) 112-117.

[2] J.W. Hoppener, B. Ahren, C.J. Lips, Islet amyloid and type 2 diabetes mellitus, N. Engl. J. Med. 343 (2000) 411-419.

[3] J. Hardy, D.J. Selkoe, The amyloid hypothesis of Alzheimer's disease: progress and problems on the road to therapeutics, Science 297 (2002) 353-356.

[4] S.P. Pallo, G.V. Johnson, Tau facilitates A $\beta$-induced loss of mitochondrial membrane potential independent of cytosolic calcium fluxes in mouse cortical neurons, Neurosci. Lett. 597 (2015) 32-37.

[5] M.A. De Anda-Hernández, K.I. Lira-De León, R. Mena, V. Campos-Peña, M.A. Meraz-Ríos, Tau and Amyloid- $\beta$ Conformational Change to $\beta$-Sheet Structures as Effectors in the Development of Alzheimer's Disease, Neuroscience - Dealing With Frontiers, 2012.

[6] G. Ramachandran, J.B. Udgaonkar, Understanding the kinetic roles of the inducer heparin and of rod-like protofibrils during amyloid fibril formation by Tau protein, J. Biol. Chem. 286 (2011) 38948-38959.

[7] Y. Wang, E. Mandelkow, Tau in physiology and pathology, Nat. Rev. Neurosci. (2015). 
[8] A.J. Lyons, N.S. Gandhi, R.L. Mancera, Molecular dynamics simulation of the phosphorylation-induced conformational changes of a Tau peptide fragment, Proteins: Structure, Function, and Bioinformatics 82 (2014) 1907-1923.

[9] E.M. Jones, M. Dubey, P.J. Camp, B.C. Vernon, J. Biernat, E. Mandelkow, J. Majewski, E.Y. Chi, Interaction of Tau protein with model lipid membranes induces Tau structural compaction and membrane disruption, Biochemistry 51 (2012) 2539-2550.

[10] S. Barghorn, E. Mandelkow, Toward a unified scheme for the aggregation of Tau into Alzheimer paired helical filaments, Biochemistry 41 (2002) 14885-14896.

[11] M.D. Mukrasch, S. Bibow, J. Korukottu, S. Jeganathan, J. Biernat, C. Griesinger, E. Mandelkow, M. Zweckstetter, Structural polymorphism of 441-residue Tau at single residue resolution, PLoS Biol. 7 (2009) e1000034.

[12] O. Karima, G. Riazi, S. Khodadadi, H. Aryapour, M.A.N. Khalili, L. Yousefi, A.A. Moosavi-Movahedi, Altered tubulin assembly dynamics with N-homocysteinylated human 1N4R Tau in vitro, FEBS Lett. 586 (2012) 3914-3919.

[13] M.A. Brister, A.K. Pandey, A.A. Bielska, N.J. Zondlo, OGlcNAcylation and phosphorylation have opposing structural effects in Tau: phosphothreonine induces particular conformational order, J. Am. Chem. Soc. 136 (2014) 3803-3816.

[14] A. Snow, H. Mar, D. Nochlin, H. Kresse, T. Wight, Peripheral distribution of dermatan sulfate proteoglycans (decorin) in amyloidcontaining plaques and their presence in neurofibrillary tangles of Alzheimer's disease, J. Histochem. Cytochem. 40 (1992) 105113.

[15] J. Su, B. Cummings, C. Cotman, Localization of heparan sulfate glycosaminoglycan and proteoglycan core protein in aged brain and Alzheimer's disease, Neuroscience 51 (1992) 801-813.

[16] A.D. Snow, H. Mar, D. Nochlin, K. Kimata, M. Kato, S. Suzuki, J. Hassell, T. Wight, The presence of heparan sulfate proteoglycans in the neuritic plaques and congophilic angiopathy in Alzheimer's disease, The American journal of pathology 133 (1988) 456.

[17] C.N. Chirita, E.E. Congdon, H. Yin, J. Kuret, Triggers of full-length Tau aggregation: a role for partially folded intermediates, Biochemistry 44 (2005) 5862-5872.

[18] E.E. Congdon, S. Kim, J. Bonchak, T. Songrug, A. Matzavinos, J. Kuret, Nucleation-dependent Tau filament formation the importance of dimerization and an estimation of elementary rate constants, J. Biol. Chem. 283 (2008) 13806-13816.

[19] S. Barghorn, P. Davies, E. Mandelkow, Tau paired helical filaments from Alzheimer's disease brain and assembled in vitro are based on beta-structure in the core domain, Biochemistry 43 (2004) 1694-1703.

[20] S. Zhu, A. Shala, A. Bezginov, A. Sljoka, G. Audette, D.J. Wilson, Hyperphosphorylation of Intrinsically Disordered Tau Protein Induces an Amyloidogenic Shift in Its Conformational Ensemble, PLoS One 10 (2015) e0120416.

[21] N. Sibille, A. Sillen, A. Leroy, J.-M. Wieruszeski, B. Mulloy, I. Landrieu, G. Lippens, Structural impact of heparin binding to fulllength Tau as studied by NMR spectroscopy, Biochemistry 45 (2006) 12560-12572.

[22] M.D. Mukrasch, J. Biernat, M. von Bergen, C. Griesinger, E. Mandelkow, M. Zweckstetter, Sites of Tau important for aggregation populate $\beta$-structure and bind to microtubules and polyanions, J. Biol. Chem. 280 (2005) 24978-24986.

[23] S.W. Carlson, M. Branden, K. Voss, Q. Sun, C.A. Rankin, T.C. Gamblin, A complex mechanism for inducer mediated Tau polymerization, Biochemistry 46 (2007) 8838-8849.

[24] M. von Bergen, S. Barghorn, S.A. Müller, M. Pickhardt, J. Biernat, E.-M. Mandelkow, P. Davies, U. Aebi, E. Mandelkow, The core of Tau-paired helical filaments studied by scanning transmission electron microscopy and limited proteolysis, Biochemistry 45 (2006) 6446-6457.

[25] O.H. Lowry, N.J. Rosebrough, A.L. Farr, R.J. Randall, Protein measurement with the Folin phenol reagent, J. Biol. Chem. 193 (1951) 265-275.

[26] G. Ramachandran, J.B. Udgaonkar, Evidence for the existence of a secondary pathway for fibril growth during the aggregation of Tau, J. Mol. Biol. 421 (2012) 296-314.

[27] L. Nielsen, R. Khurana, A. Coats, S. Frokjaer, J. Brange, S. Vyas, V.N. Uversky, A.L. Fink, Effect of environmental factors on the kinetics of insulin fibril formation: elucidation of the molecular mechanism, Biochemistry 40 (2001) 6036-6046.

[28] R. Yazdanparast, R. Khodarahmi, E. Soori, Comparative studies of the artificial chaperone-assisted refolding of thermally denatured bovine carbonic anhydrase using different capturing ionic detergents and $\beta$-cyclodextrin, Arch. Biochem. Biophys. 437 (2005) $178-185$.

[29] M.J. Abraham, T. Murtola, R. Schulz, S. Páll, J.C. Smith, B. Hess, E. Lindahl, GROMACS: High performance molecular simulations through multi-level parallelism from laptops to supercomputers, SoftwareX 1 (2015) 19-25.

[30] S.R. Comeau, D.W. Gatchell, S. Vajda, C.J. Camacho, ClusPro: an automated docking and discrimination method for the prediction of protein complexes, Bioinformatics 20 (2004) 45-50.

[31] C.M. Torres-Bugeau, C.L. Ávila, R. Raisman-Vozari, D. Papy-Garcia, R. Itri, L.R. Barbosa, L.M. Cortez, V.L. Sim, R.N. Chehín, Characterization of heparin-induced glyceraldehyde-3-phosphate dehydrogenase early amyloid-like oligomers and their implication in $\alpha$-synuclein aggregation, J. Biol. Chem. 287 (2012) 2398-2409.

[32] W. Humphrey, A. Dalke, K. Schulten, VMD: visual molecular dynamics, J. Mol. Graphics 14 (1996) 33-38.

[33] M. Robert, P. Mathuranath, Tau and Tauopathies, Neurol. India 55 (2007) 11.

[34] L. Martin, X. Latypova, F. Terro, Post-translational modifications of Tau protein: implications for Alzheimer's disease, Neurochem. Int. 58 (2011) 458-471.

[35] F. Chiti, C.M. Dobson, Protein misfolding, functional amyloid, and human disease, Annu. Rev. Biochem. 75 (2006) $333-366$.

[36] S. Vilasi, R. Sarcina, R. Maritato, A. De Simone, G. Irace, I. Sirangelo, Heparin induces harmless fibril formation in amyloidogenic W7FW14F apomyoglobin and amyloid aggregation in wild-type protein in vitro, PLoS One 6 (2011) e22076.

[37] A. Relini, S. De Stefano, S. Torrassa, O. Cavalleri, R. Rolandi, A. Gliozzi, S. Giorgetti, S. Raimondi, L. Marchese, L. Verga, Heparin strongly enhances the formation of $\beta 2$-microglobulin amyloid fibrils in the presence of type I collagen, J. Biol. Chem. 283 (2008) 4912-4920. 
[38] M. Hasegawa, R.A. Crowther, R. Jakes, M. Goedert, Alzheimer-like changes in microtubule-associated protein Tau induced by sulfated glycosaminoglycans Inhibition of microtubule binding, stimulation of phosphorylation, and filament assembly depend on the degree of sulfation, J. Biol. Chem. 272 (1997) 33118-33124.

[39] R.G. Ingle, A.S. Agarwal, A world of low molecular weight heparins (LMWHs) enoxaparin as a promising moiety-A review, Carbohydr. Polym. 106 (2014) 148-153.

[40] J. Kuret, C.N. Chirita, E.E. Congdon, T. Kannanayakal, G. Li, M. Necula, H. Yin, Q. Zhong, Pathways of Tau fibrillization, Biochim. Biophys. Acta 1739 (2005) 167-178.

[41] L.-w. Ko, M. DeTure, N. Sahara, R. Chihab, I.E. Vega, S.-H. Yen, Recent advances in experimental modeling of the assembly of Tau filaments, Biochim. Biophys. Acta 1739 (2005) 125-139.

[42] N. Sahara, S. Maeda, M. Murayama, T. Suzuki, N. Dohmae, S.H. Yen, A. Takashima, Assembly of two distinct dimers and higher-order oligomers from full-length Tau, Eur. J. Neurosci. 25 (2007) 3020-3029.

[43] H.-L. Zhu, C. Fernández, J.-B. Fan, F. Shewmaker, J. Chen, A.P. Minton, Y. Liang, Quantitative characterization of heparin binding to Tau protein Implication for inducer-mediated Tau filament formation, J. Biol. Chem. 285 (2010) 3592-3599.

[44] S.B. Nielsen, P. Yde, L. Giehm, S. Sundbye, G. Christiansen, J. Mathiesen, M.H. Jensen, P.H. Jensen, D.E. Otzen, Multiple Roles of Heparin in the Aggregation of p25 $\alpha$, J. Mol. Biol. 421 (2012) 601-615.

[45] M. Von Bergen, S. Barghorn, J. Biernat, E.-M. Mandelkow, E. Mandelkow, Tau aggregation is driven by a transition from random coil to beta sheet structure, Biochim. Biophys. Acta 1739 (2005) 158-166.

[46] R. Khodarahmi, M. Beyrami, H. Soori, Appraisal of casein's inhibitory effects on aggregation accompanying carbonic anhydrase refolding and heat-induced ovalbumin fibrillogenesis, Arch. Biochem. Biophys. 477 (2008) 67-76.

[47] N. Rezaei-Ghaleh, A. Ebrahim-Habibi, A.A. Moosavi-Movahedi, M. Nemat-Gorgani, Role of electrostatic interactions in 2, 2, 2trifluoroethanol-induced structural changes and aggregation of $\alpha$-chymotrypsin, Arch. Biochem. Biophys. 457 (2007) $160-169$.

[48] M. Fändrich, Absolute correlation between lag time and growth rate in the spontaneous formation of several amyloid-like aggregates and fibrils, J. Mol. Biol. 365 (2007) 1266-1270.

[49] V.A. Borzova, K.A. Markossian, B.I. Kurganov, Relationship between the initial rate of protein aggregation and the lag period for amorphous aggregation, Int. J. Biol. Macromol. 68 (2014) 144-150.

[50] B. Kurganov, Kinetics of protein aggregation. Quantitative estimation of the chaperone-like activity in test-systems based on suppression of protein aggregation, Biochemistry (Moscow) 67 (2002) 409-422.

[51] C. Cantor, P. Schimmel, Biophysical Chemistry Part 1, The Conformation of Biological MacromoleculesFreeman, New York (1980) 115-116.

[52] H. Shoval, L. Weiner, E. Gazit, M. Levy, I. Pinchuk, D. Lichtenberg, Polyphenol-induced dissociation of various amyloid fibrils results in a methionine-independent formation of ROS, Biochimica et Biophysica Acta (BBA)-Proteins and Proteomics 1784 (2008) 1570-1577.

[53] R. Khodarahmi, H. Soori, M. Amani, Study of Cosolvent-Induced $\alpha$-Chymotrypsin Fibrillogenesis: Does Protein Surface Hydrophobicity Trigger Early Stages of Aggregation Reaction?, Protein J. 28 (2009) 349-361.

[54] S. Jeganathan, M. von Bergen, E.-M. Mandelkow, E. Mandelkow, The Natively Unfolded Character of Tau and Its Aggregation to Alzheimer-like Paired Helical Filaments†, Biochemistry 47 (2008) 10526-10539.

[55] K. Flach, I. Hilbrich, A. Schiffmann, U. Gärtner, M. Krüger, M. Leonhardt, H. Waschipky, L. Wick, T. Arendt, M. Holzer, Tau oligomers impair artificial membrane integrity and cellular viability, J. Biol. Chem. 287 (2012) 43223-43233.

[56] A. Fresht, Structure and mechanism in protein science: a guide to enzyme catalysis and protein folding, WH Freeman and Co., New York, 1999.

[57] C.N. Chirita, J. Kuret, Evidence for an intermediate in Tau filament formation, Biochemistry 43 (2004) 1704-1714.

[58] J. Kuret, E.E. Congdon, G. Li, H. Yin, X. Yu, Q. Zhong, Evaluating triggers and enhancers of Tau fibrillization, Microsc. Res. Tech. 67 (2005) 141-155.

[59] S.M. Hartig, Basic image analysis and manipulation in ImageJ, Curr. Protoc. Mol. Biol. (2013) 14.15. 11-14.15. 12.

[60] M. von Bergen, S. Barghorn, L. Li, A. Marx, J. Biernat, E.-M. Mandelkow, E. Mandelkow, Mutations of Tau protein in frontotemporal dementia promote aggregation of paired helical filaments by enhancing local $\beta$-structure, J. Biol. Chem. 276 (2001) 48165-48174.

[61] A. Perálvarez-Marín, L. Mateos, C. Zhang, S. Singh, Á. Cedazo-Mínguez, N. Visa, L. Morozova-Roche, A. Gräslund, A. Barth, Influence of residue 22 on the folding, aggregation profile, and toxicity of the Alzheimer's amyloid $\beta$ peptide, Biophys. J. 97 (2009) 277-285.

[62] O.S. Makin, L.C. Serpell, X-ray diffraction studies of amyloid structure, Amyloid proteins: methods and protocols (2005) 67-80.

[63] S. Banerjee, S. Maity, A.S. Chakraborti, Methylglyoxal-induced modification causes aggregation of myoglobin, Spectrochimica Acta Part A: Molecular and Biomolecular Spectroscopy 155 (2016) 1-10.

[64] T. Lührs, C. Ritter, M. Adrian, D. Riek-Loher, B. Bohrmann, H. Döbeli, D. Schubert, R. Riek, 3D structure of Alzheimer's amyloid$\beta(1-42)$ fibrils, Proc. Natl. Acad. Sci. U. S. A. 102 (2005) 17342-17347.

[65] D.M. Fowler, A.V. Koulov, W.E. Balch, J.W. Kelly, Functional amyloid-from bacteria to humans, Trends Biochem. Sci. 32 (2007) 217-224.

[66] K.L. Morris, L.C. Serpell, X-ray fibre diffraction studies of amyloid fibrils, Springer, 2012.

[67] O.S. Makin, E. Atkins, P. Sikorski, J. Johansson, L.C. Serpell, Molecular basis for amyloid fibril formation and stability, Proc. Natl. Acad. Sci. U. S. A. 102 (2005) 315-320.

[68] H. Inouye, D. Sharma, W.J. Goux, D.A. Kirschner, Structure of core domain of fibril-forming PHF/Tau fragments, Biophys. J. 90 (2006) 1774-1789. 
[69] C. Lara, S. Gourdin-Bertin, J. Adamcik, S. Bolisetty, R. Mezzenga, Self-assembly of ovalbumin into amyloid and non-amyloid fibrils, Biomacromolecules 13 (2012) 4213-4221.

[70] A. Naeem, S. Amani, Deciphering structural intermediates and genotoxic fibrillar aggregates of albumins: a molecular mechanism underlying for degenerative diseases, PLoS One 8 (2013) e54061.

[71] N.K. Holm, S.K. Jespersen, L.V. Thomassen, T.Y. Wolff, P. Sehgal, L.A. Thomsen, G. Christiansen, C.B. Andersen, A.D. Knudsen, D.E. Otzen, Aggregation and fibrillation of bovine serum albumin, Biochim. Biophys. Acta 1774 (2007) 1128-1138.

[72] M.D. Mukrasch, M. von Bergen, J. Biernat, D. Fischer, C. Griesinger, E. Mandelkow, M. Zweckstetter, The "jaws" of the Taumicrotubule interaction, J. Biol. Chem. 282 (2007) 12230-12239.

[73] M. Hennig, B. Darimont, R. Sterner, K. Kirschner, J.N. Jansonius, 2.0 A structure of indole-3-glycerol phosphate synthase from the hyperthermophile Sulfolobus solfataricus: possible determinants of protein stability, Structure 3 (1995) 1295-1306.

[74] R. Fraczkiewicz, W. Braun, Exact and efficient analytical calculation of the accessible surface areas and their gradients for macromolecules, J. Comput. Chem. 19 (1998) 319-333.

[75] J.Y. Suk, F. Zhang, W.E. Balch, R.J. Linhardt, J.W. Kelly, Heparin accelerates gelsolin amyloidogenesis, Biochemistry 45 (2006) 2234-2242.

[76] S. Elbaum-Garfinkle, E. Rhoades, Identification of an aggregation-prone structure of Tau, J. Am. Chem. Soc. 134 (2012) 1660716613.

[77] M. Lyon, J.T. Gallagher, Bio-specific sequences and domains in heparan sulphate and the regulation of cell growth and adhesion, Matrix Biol. 17 (1998) 485-493.

[78] P. Vongchan, M. Warda, H. Toyoda, T. Toida, R.M. Marks, R.J. Linhardt, Structural characterization of human liver heparan sulfate, Biochim. Biophys. Acta 1721 (2005) 1-8.

[79] L.O. Sampaio, I.L. Tersariol, C.C. Lopes, R.I. Bouças, F.D. Nascimento, H.A. Rocha, H.B. Nader, Heparins and heparans sulfates. Structure, distribution and protein interactions, Insights into carbohydrate structure and biological function. Kerala: Transworld Research Network (2006) 51-61. 
Table 1. Kinetic parameters of heparin concentration on Tau fibril formation. Kinetic traces of Tau fibril formation are shown in Fig. 3 and fitted to the sigmoidal function (see Equation 1). Errors are estimated by two or more independent experiments.

\begin{tabular}{cccc}
\hline Heparin & $\boldsymbol{k}_{\text {app }}$ & $\boldsymbol{t}_{\mathrm{D}}$ & $\boldsymbol{F}_{\max }$ \\
\hline $\mathbf{\mu M}$ & $\mathrm{h}^{-1}$ & $\mathrm{~h}$ & \\
$\mathbf{9 . 5}$ & $(10.53 \pm 1.09) \times 10^{-2}$ & $16.24 \pm 1.07$ & $(31.55 \pm 1.93) \times 10$ \\
$\mathbf{1 2}$ & $(16.71 \pm 1.64) \times 10^{-2}$ & $12.53 \pm 0.76$ & $(47.16 \pm 1.68) \times 10$ \\
$\mathbf{1 6}$ & $(21.47 \pm 1.28) \times 10^{-2}$ & $9.41 \pm 0.73$ & $(63.12 \pm 2.17) \times 10$ \\
$\mathbf{2 2}$ & $(16.76 \pm 1.41) \times 10^{-2}$ & $11.08 \pm 1.13$ & $(59.12 \pm 1.95) \times 10$ \\
$\mathbf{3 3}$ & $(12.11 \pm 0.59) \times 10^{-2}$ & $14.94 \pm 1.27$ & $(43.64 \pm 1.83) \times 10$ \\
$\mathbf{4 4}$ & $(8.07 \pm 0.47) \times 10^{-2}$ & $19.13 \pm 0.84$ & $(17.58 \pm 1.91) \times 10$ \\
\hline
\end{tabular}

Table 2. Kinetic parameters of enoxaparin concentration on Tau fibril formation. Kinetic traces of Tau fibril formation are shown in Fig. 3 and fitted to the sigmoidal function (see Equation 1). Errors are estimated by two or more independent experiments.

\begin{tabular}{cccc}
\hline Enoxaparin & $\boldsymbol{k}_{\text {app }}$ & $\boldsymbol{t}_{\mathrm{D}}$ & $\boldsymbol{F}_{\text {max }}$ \\
\hline $\boldsymbol{\mu M}$ & $\mathrm{h}^{-1}$ & $\mathrm{~h}$ & \\
$\mathbf{3 9}$ & $(7.77 \pm 0.48) \times 10^{-2}$ & $18.06 \pm 1.39$ & $(6.95 \pm 1.39) \times 10$ \\
$\mathbf{5 1}$ & $(11.41 \pm 1.01) \times 10^{-2}$ & $13.34 \pm 1.04$ & $(13.21 \pm 1.04) \times 10$ \\
$\mathbf{6 3}$ & $(15.32 \pm 1.13) \times 10^{-2}$ & $11.41 \pm 0.86$ & $(23.46 \pm 1.67) \times 10$ \\
$\mathbf{1 0 0}$ & $(13.62 \pm 0.41) \times 10^{-2}$ & $13.18 \pm 1.23$ & $(16.89 \pm 1.27) \times 10$ \\
$\mathbf{1 3 7}$ & $(9.43 \pm 0.39) \times 10^{-2}$ & $18.11 \pm 0.99$ & $(10.31 \pm 1.42) \times 10$ \\
$\mathbf{1 7 4}$ & $(5.92 \pm 1.24) \times 10^{-2}$ & $22.46 \pm 1.08$ & $(5.08 \pm 1.76) \times 10$ \\
\hline
\end{tabular}


Table 3. Kinetic parameters of Tau concentration on Tau fibril formation in the presence of $16 \mu \mathrm{M}$ heparin. Kinetic traces of Tau fibril formation are shown in Fig. 6 and fitted to the sigmoidal function (see Equation 1). Errors are estimated by two or more independent experiments.

\begin{tabular}{cccc}
\hline Tau & $\boldsymbol{k}_{\text {app }}$ & $\boldsymbol{t}_{\mathrm{D}}$ & $\boldsymbol{F}_{\max }$ \\
\hline $\boldsymbol{\mu M}$ & $\mathrm{h}^{-1}$ & $\mathrm{~h}$ & \\
$\mathbf{1 6}$ & $(5.03 \pm 0.35) \times 10^{-2}$ & $19.63 \pm 1.07$ & $(12.11 \pm 1.88) \times 10$ \\
$\mathbf{2 2}$ & $(10.11 \pm 0.87) \times 10^{-2}$ & $15.53 \pm 0.87$ & $(23.57 \pm 1.91) \times 10$ \\
$\mathbf{3 6}$ & $(14.55 \pm 0.52) \times 10^{-2}$ & $12.28 \pm 0.99$ & $(43.46 \pm 1.95) \times 10$ \\
$\mathbf{5 8}$ & $(21.47 \pm 1.28) \times 10^{-2}$ & $9.41 \pm 0.73$ & $(63.12 \pm 2.17) \times 10$ \\
$\mathbf{7 3}$ & $(15.61 \pm 0.69) \times 10^{-2}$ & $14.28 \pm 1.17$ & $(54.03 \pm 2.26) \times 10$ \\
$\mathbf{8 8}$ & $(10.32 \pm 0.76) \times 10^{-2}$ & $19.47 \pm 1.25$ & $(34.21 \pm 2.14) \times 10$ \\
$\mathbf{9 5}$ & $(6.57 \pm 0.52) \times 10^{-2}$ & $23.81 \pm 1.04$ & $(14.51 \pm 1.83) \times 10$ \\
\hline
\end{tabular}

Table 4. Kinetic parameters of Tau concentration on Tau fibril formation in the presence of $63 \mu \mathrm{M}$ enoxaparin. Kinetic traces of Tau fibril formation are shown in Fig. 6 and fitted to the sigmoidal function (see Equation 1). Errors are estimated by two or more independent experiments.

\begin{tabular}{cccc}
\hline Tau & $\boldsymbol{k}_{\text {app }}$ & $\boldsymbol{t}_{\mathrm{D}}$ & $\boldsymbol{F}_{\max }$ \\
\hline $\boldsymbol{\mu M}$ & $\mathrm{h}^{-1}$ & $\mathrm{~h}$ & \\
$\mathbf{1 6}$ & $(6.84 \pm 0.85) \times 10^{-2}$ & $20.09 \pm 1.25$ & $(7.51 \pm 1.02) \times 10$ \\
$\mathbf{2 2}$ & $(10.62 \pm 0.82) \times 10^{-2}$ & $17.03 \pm 1.23$ & $(11.64 \pm 1.23) \times 10$ \\
$\mathbf{3 6}$ & $(13.86 \pm 0.79) \times 10^{-2}$ & $14.28 \pm 0.74$ & $(18.02 \pm 1.38) \times 10$ \\
$\mathbf{5 8}$ & $(15.32 \pm 1.13) \times 10^{-2}$ & $11.41 \pm 0.86$ & $(23.46 \pm 1.67) \times 10$ \\
$\mathbf{7 3}$ & $(11.47 \pm 0.67) \times 10^{-2}$ & $15.28 \pm 0.93$ & $(21.87 \pm 2.03) \times 10$ \\
$\mathbf{8 8}$ & $(8.65 \pm 0.58) \times 10^{-2}$ & $19.97 \pm 1.02$ & $(14.95 \pm 1.72) \times 10$ \\
$\mathbf{9 5}$ & $(6.88 \pm 0.62) \times 10^{-2}$ & $24.11 \pm 1.19$ & $(7.76 \pm 1.49) \times 10$ \\
\hline
\end{tabular}


Table 5. Calculation of ASA for two key aggregation sites of Tau using GETAREA.

\begin{tabular}{|c|c|c|c|c|c|c|}
\hline \multicolumn{7}{|c|}{ Accessibility Surface Area (ASA) } \\
\hline \multirow[b]{2}{*}{$\begin{array}{l}\text { Aggregation-prone } \\
\text { Segment I }\end{array}$} & \multicolumn{3}{|c|}{ Single Tau } & \multicolumn{3}{|c|}{ Tau-heparin complex } \\
\hline & Total & Backbone & Side chain & Total & Backbone & Side chain \\
\hline K274 & 71.36 & 54.59 & 12.77 & 74.55 & 64.08 & 17.75 \\
\hline V275 & 21.88 & 21.16 & 0.79 & 26 & 25.33 & 0.76 \\
\hline Q276 & 9.11 & 3.51 & 0.12 & 9.24 & 4.49 & 0.16 \\
\hline I277 & 17.3 & 17.28 & 0.02 & 23.61 & 23.61 & 0 \\
\hline $\mathbf{I 2 7 8}$ & 1.72 & 1.71 & 0.01 & 2.9 & 2.9 & 0.2 \\
\hline N279 & 8.12 & 4.62 & 3.58 & 11.35 & 7.81 & 4.28 \\
\hline K280 & 11.04 & 4.7 & 5.45 & 13.61 & 8.19 & 5.07 \\
\hline \multicolumn{7}{|l|}{ Aggregation-prone } \\
\hline V306 & 4.01 & 4.01 & 0.01 & 7.66 & 7.66 & 0.01 \\
\hline Q307 & 16.21 & 3.64 & 0.01 & 16.39 & 8.75 & 0.04 \\
\hline I308 & 10.81 & 9.66 & 1.15 & 17.3 & 13.69 & 3.61 \\
\hline V309 & 2.73 & 2.73 & 2.37 & 5.36 & 5.36 & 3.14 \\
\hline Y310 & 92.14 & 75.57 & 9.63 & 121.15 & 91.73 & 11.08 \\
\hline K311 & 125.88 & 89.42 & 1.28 & 130.28 & 102.52 & 0.49 \\
\hline
\end{tabular}



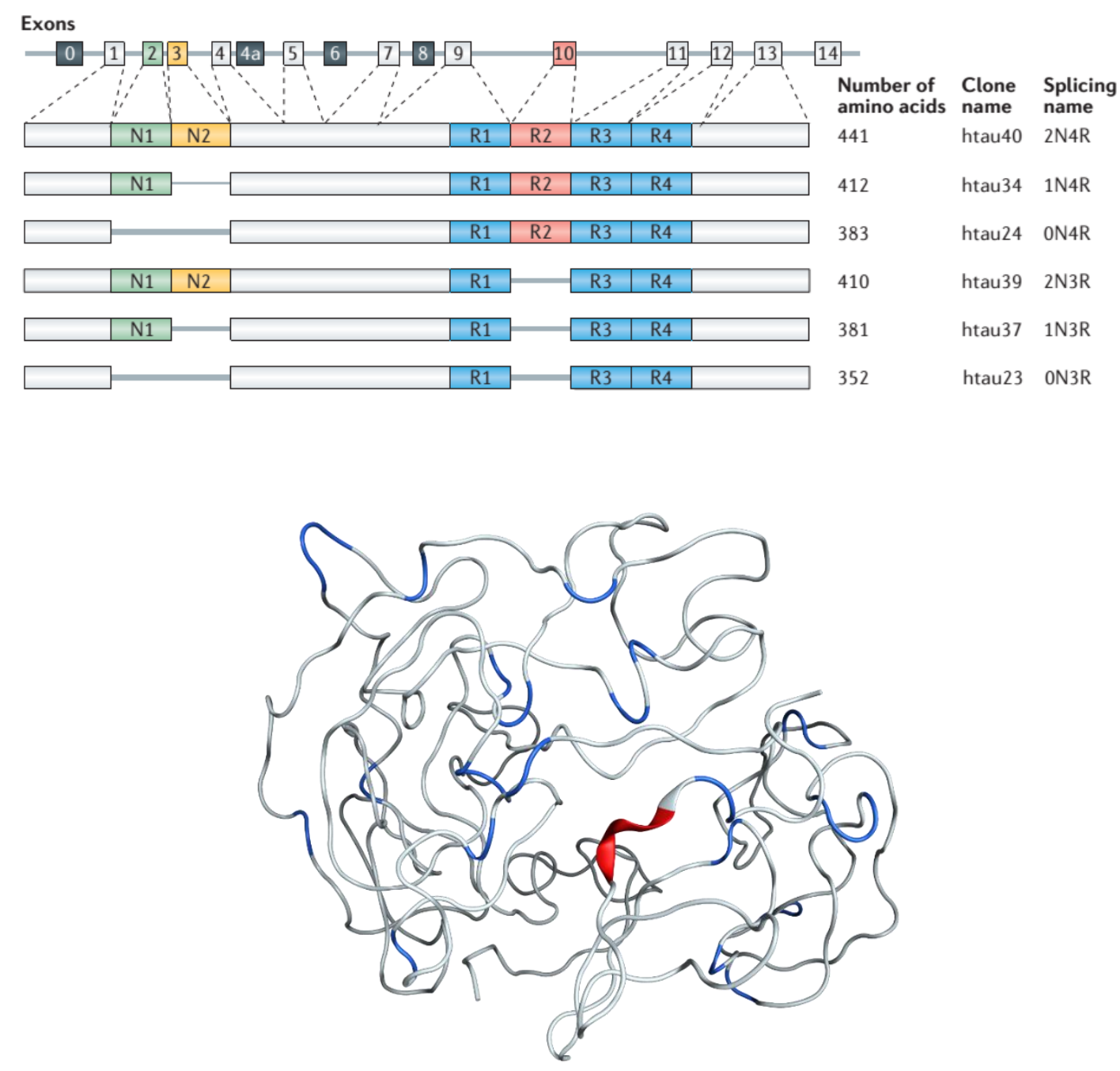

Fig. 1. TOP The human microtubule-associated protein Tau gene (MAPT) gene and the splice isoforms of Tau in the human brain. MAPT, the gene encoding human Tau, contains 16 exons. Some of them are constitutive, whereas the others are subject to alternative splicing. The six human brain Tau isoforms are generated through alternative splicing of E2, E3 and E10. These Tau isoforms differ according to the presence of 0,1 or 2 near-amino-terminal inserts $(0 \mathrm{~N}, 1 \mathrm{~N}$ or $2 \mathrm{~N}$, respectively) and the presence of repeat R2, yielding 3 or 4 carboxy-terminal repeat domain (3R or 4R, respectively) Tau species. The expression of human Tau is developmentally regulated: in the adult brain, six isoforms of Tau are expressed and the levels of the $3 \mathrm{R}$ and $4 \mathrm{R}$ forms are roughly equal and the $2 \mathrm{~N}$ isoform is underrepresented compared with the others: the $0 \mathrm{~N}, 1 \mathrm{~N}$ and $2 \mathrm{~N}$ Tau isoforms comprise $\sim 37 \%, \sim 54 \%$ and $\sim 9 \%$ of total Tau, respectively. The expression of Tau in the human brain also shows considerable regional variation. The mRNA and protein levels of Tau in the neocortex are twofold higher than those in the white matter and cerebellum. This may contribute to the differential vulnerability of brain regions to Tau pathology, Figure and caption are taken from [18]. Bottom The "most representative" structure of the native ensemble of 441-residue Tau, initialized baed on PDB coordinates provided in [11], taken from [20]. This graphical representation was depicted by VMD version 19.2. 

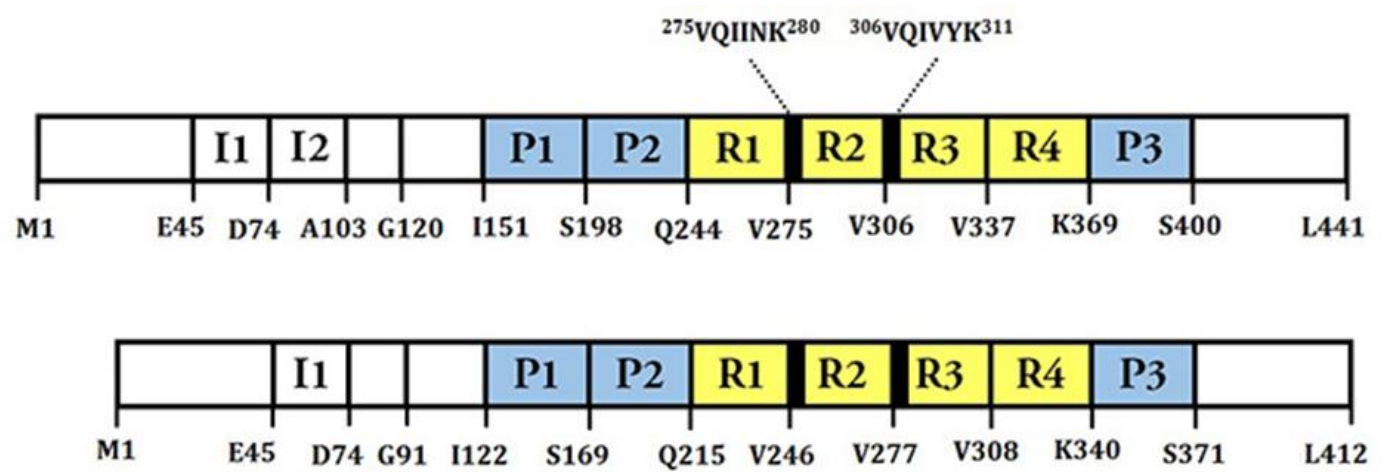

Fig. 2. Schematic representation diagram of two human Tau isoforms hT40 (2N4R) and hT34 (1N4R). Fulllength adult Tau (2N4R) contains 441 residues and can be subdivided into several domains [프. The Nterminal "projection domain" comprises residues 1-197 and does not contribute to microtubule binding. The C-terminal "assembly domain" contains the residues important for microtubule nucleation and stabilization. The four repeats (31 or 32 residues) are denoted as R1-R4. The two hexapeptide motifs ${ }^{275} \mathrm{VQIINK}^{280}$ and ${ }^{306} \mathrm{VQIVYK}^{311}$ in R2 and R3, which are necessary for PHF formation [22], are indicated by black rectangles. The 1N4R isoform is lacking 29 amino acids in the projection domain. 

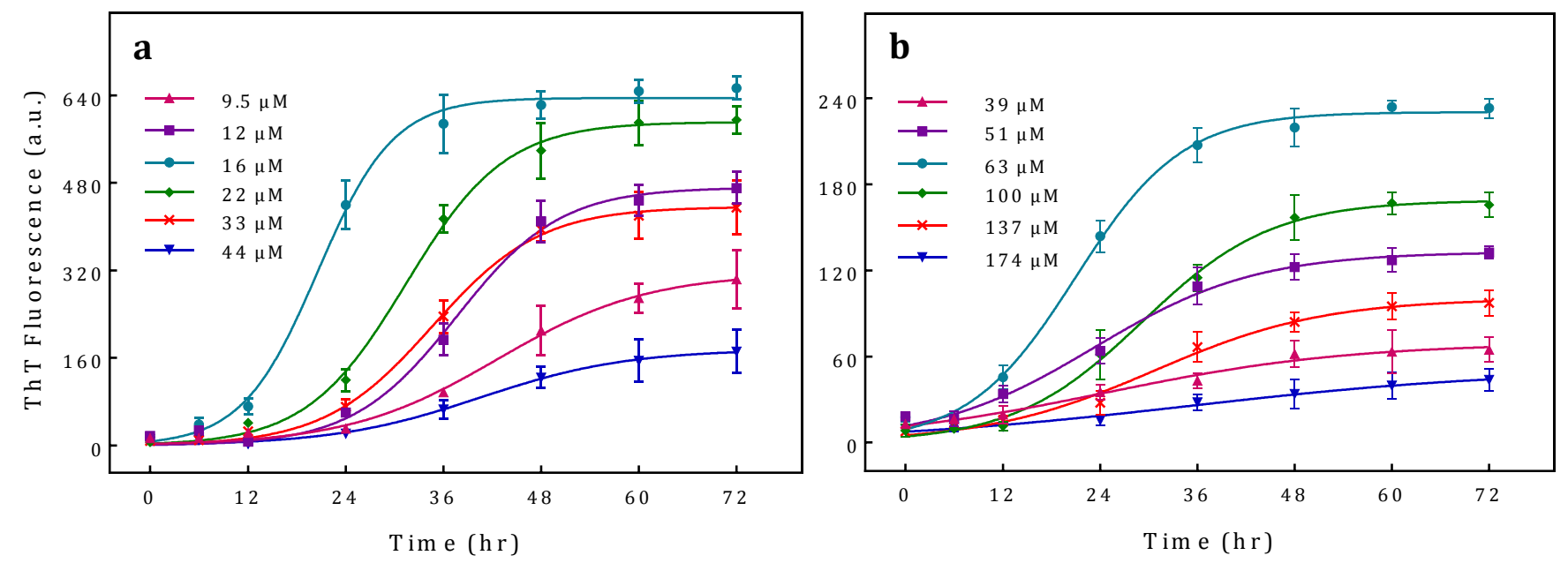

Fig. 3. Kinetic effects of heparin concentration on Tau fibril formation. ThT fluorescence monitored kinetics of $58 \mu \mathrm{M}$ Tau in the presence of various concentrations of heparin (a) and enoxaparin (b) at $37{ }^{\circ} \mathrm{C}$ in $50 \mathrm{mM}$ Tris- $\mathrm{HCl}$ buffer, $\mathrm{pH}$ 7.6, and $5 \mathrm{mM}$ DTT. The continuous line through the data points in the figure is a least square fit to a sigmoidal equation. The error bars represent the spread in the data calculated from two or more independent experiments. 

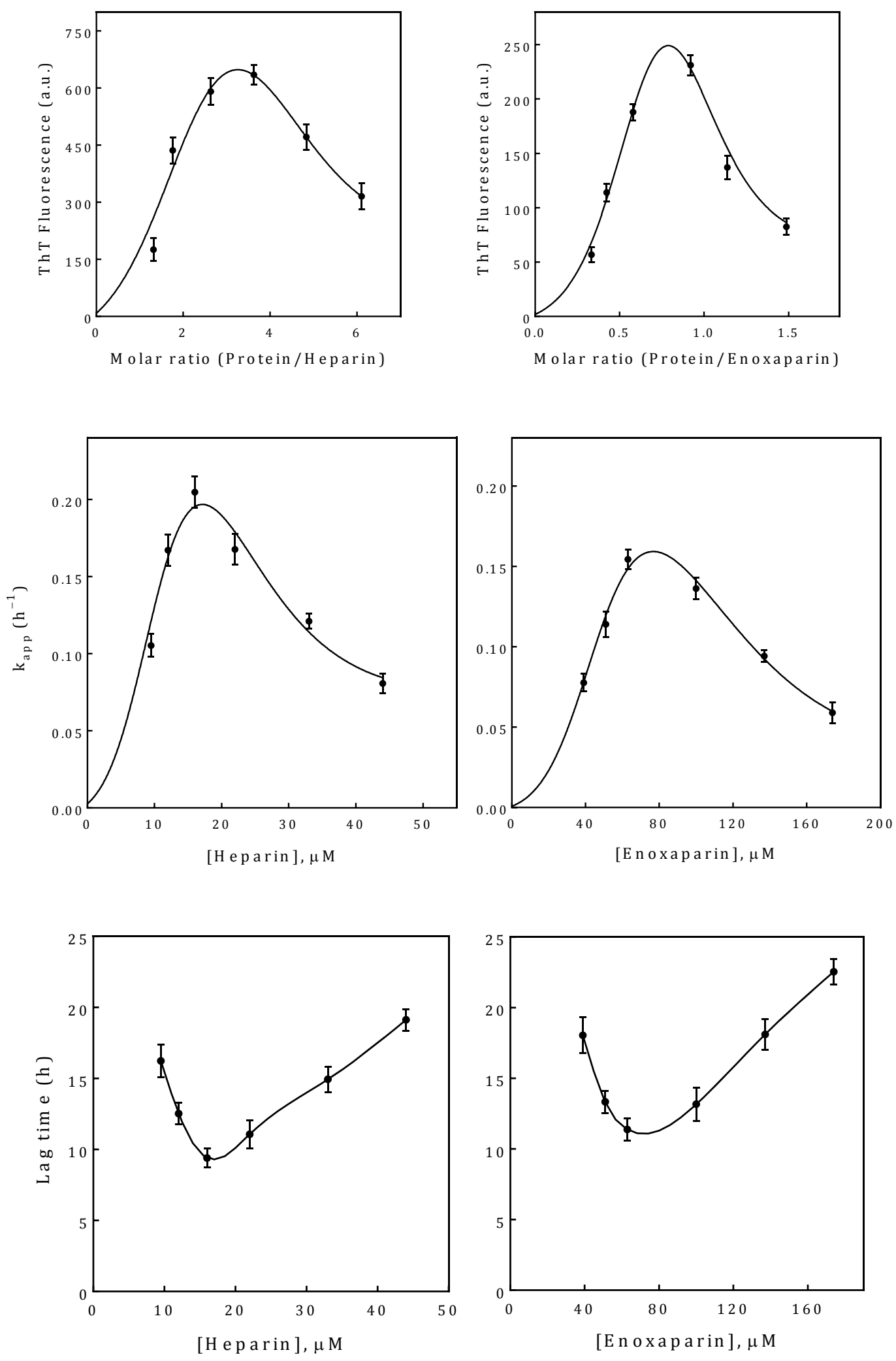
Fig. 4. (Top) The amplitude of change in ThT fluorescence is plotted against the molar ratio of protein to the inducer. The same observations were also made for Dependency of the final ThT fluorescence (aggregation extent) on the heparin and enoxaparin concentration. (Middle) Dependence of the apparent rate constant of ThT fluorescence (Tau aggregation rate) on the inducer concentration. Final ThT fluorescence is plotted as a function of molar ratio from aggregation experiments wherein the protein concentration is held constant and the inducers concentration are varied. (Bottom) Dependence of the lag time of ThT fluorescence monitored kinetics on inducers concentration. The lag time of ThT-monitored kinetics is plotted against heparin (Left) and enoxaparin (Right) concentration. Extrapolation from the linear part of sigmoidal curves (time constant $\left(t_{i}\right)$ ) to the abscissa intercept yields lag time, which is inversely proportional to nucleation rate. The error bars represent the spread in the data calculated from two or more independent experiments. 

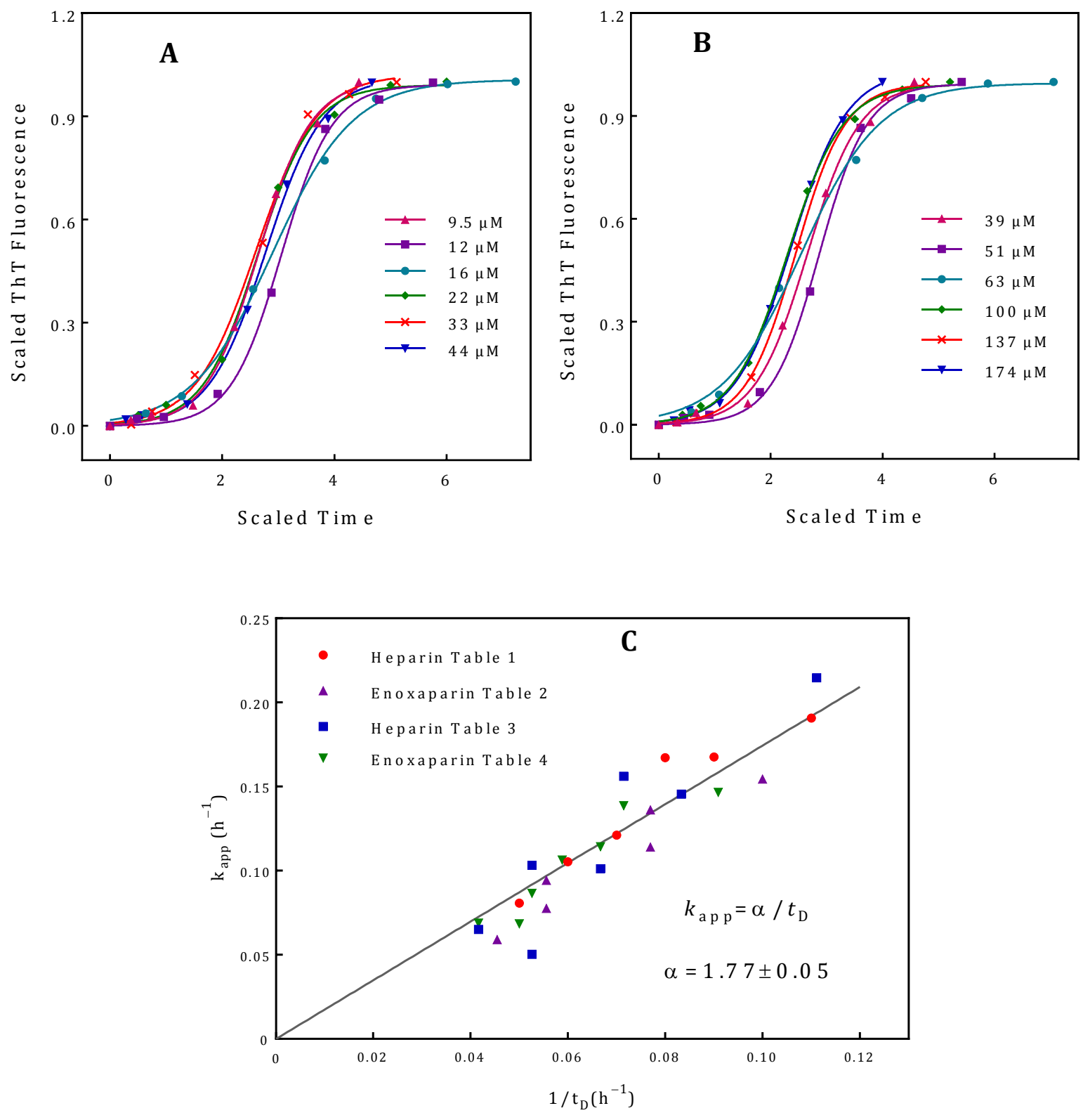

Fig. 5. Re-plotting the Kinetic effects of heparin (A) and enoxaparin (B) concentration on Tau fibril formation data after scaling ThT fluorescence as $\mathrm{F} / \mathrm{F}_{\max }$ and time as $\mathrm{t} / \mathrm{t}_{\mathrm{D}}$. The collapse of data indicates that the underlying aggregation mechanism is relatively simple. (C) Growth rate and Lag time correlation. Heparininduced aggregated samples, at all concentrations, possess kinetic properties similar to those samples induced by enoxaparin. 

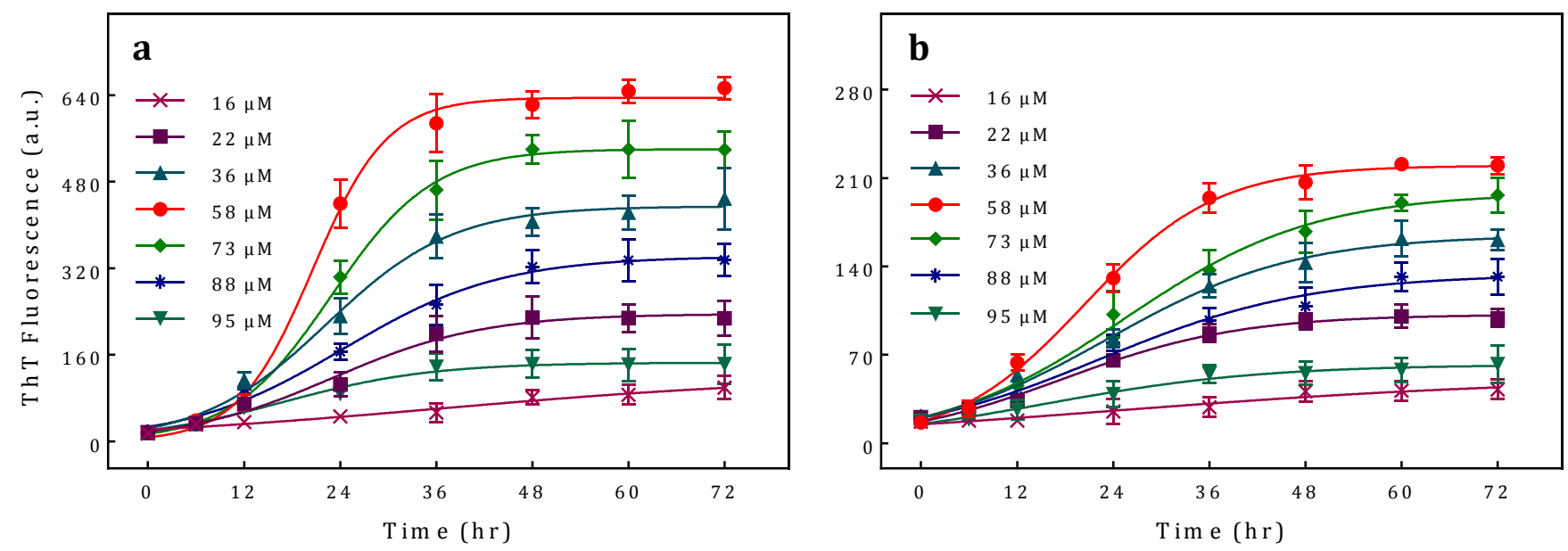

Fig. 6. Kinetic effects of protein concentration on Tau fibril formation. Kinetics of fibril formation was followed with ThT fluorescence by various concentrations of Tau in the presence of $16 \mu \mathrm{M}$ heparin (a) and 63 $\mu \mathrm{M}$ enoxaparin (b) at $37{ }^{\circ} \mathrm{C}$ in $50 \mathrm{mM}$ Tris- $\mathrm{HCl}$ buffer, $\mathrm{pH}$ 7.6, and $5 \mathrm{mM}$ DTT. The continuous lines through the data points are least-squares fits to a sigmoidal equation. The error bars represent the spread in the data calculated from two or more independent experiments.
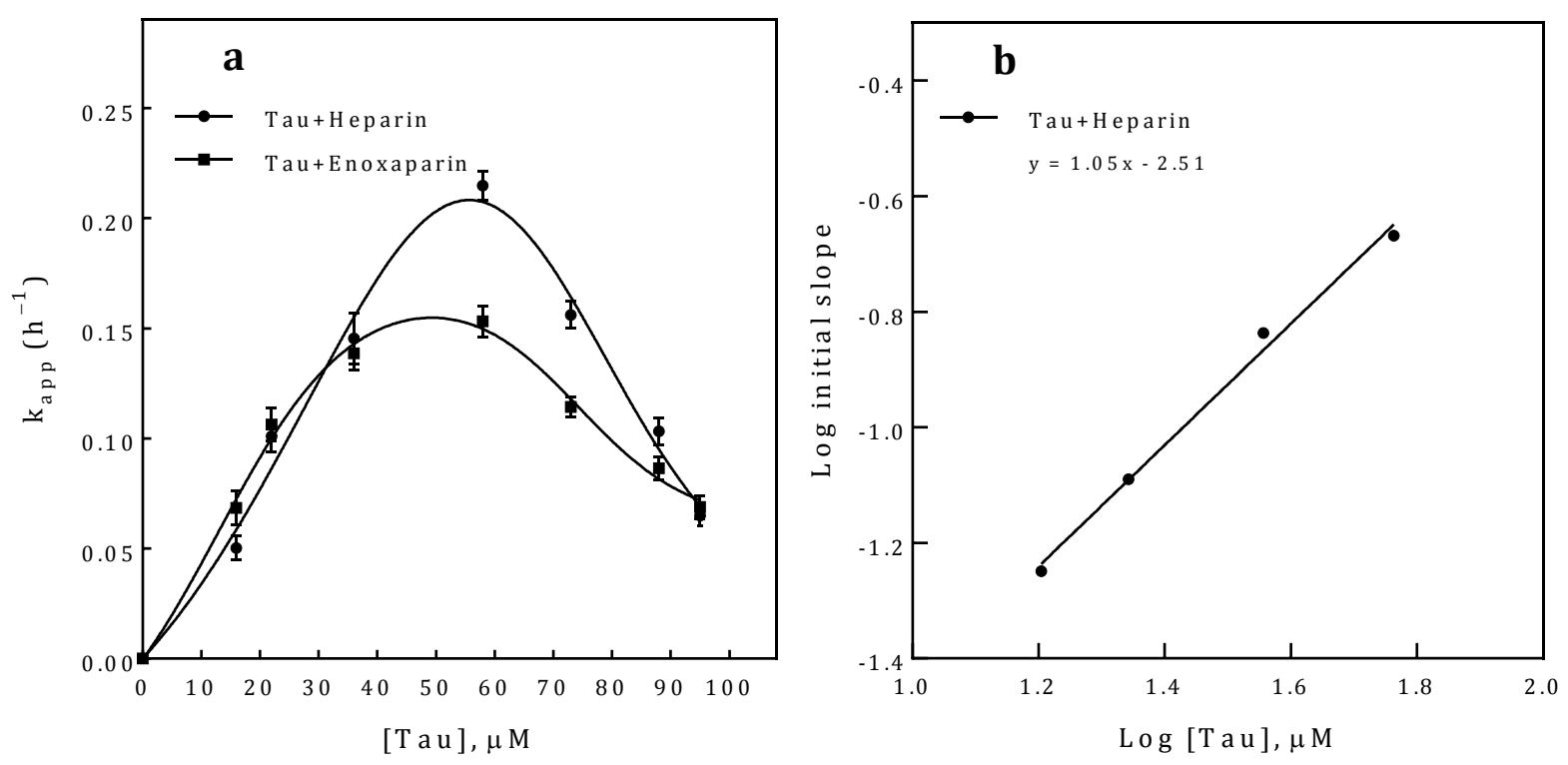

Fig. 7. (a) Dependence of the apparent rate constant of ThT fluorescence monitored kinetics on Tau concentration in the present of $16 \mu \mathrm{M}$ heparin and $63 \mu \mathrm{M}$ enoxaparin. The error bars represent the spread in the data calculated from two or more independent experiments. (b) Protomer intermediates formation is first order with respect to Tau concentration. Replotting of the initial velocities estimated from the progress curves versus Tau concentration in double logarithmic format, where the line represents the best fit of the data points to a linear regression. The slope of the replot was consistent with the initial velocity of ThT signal generation being directly proportional to Tau concentration under these experimental conditions. 


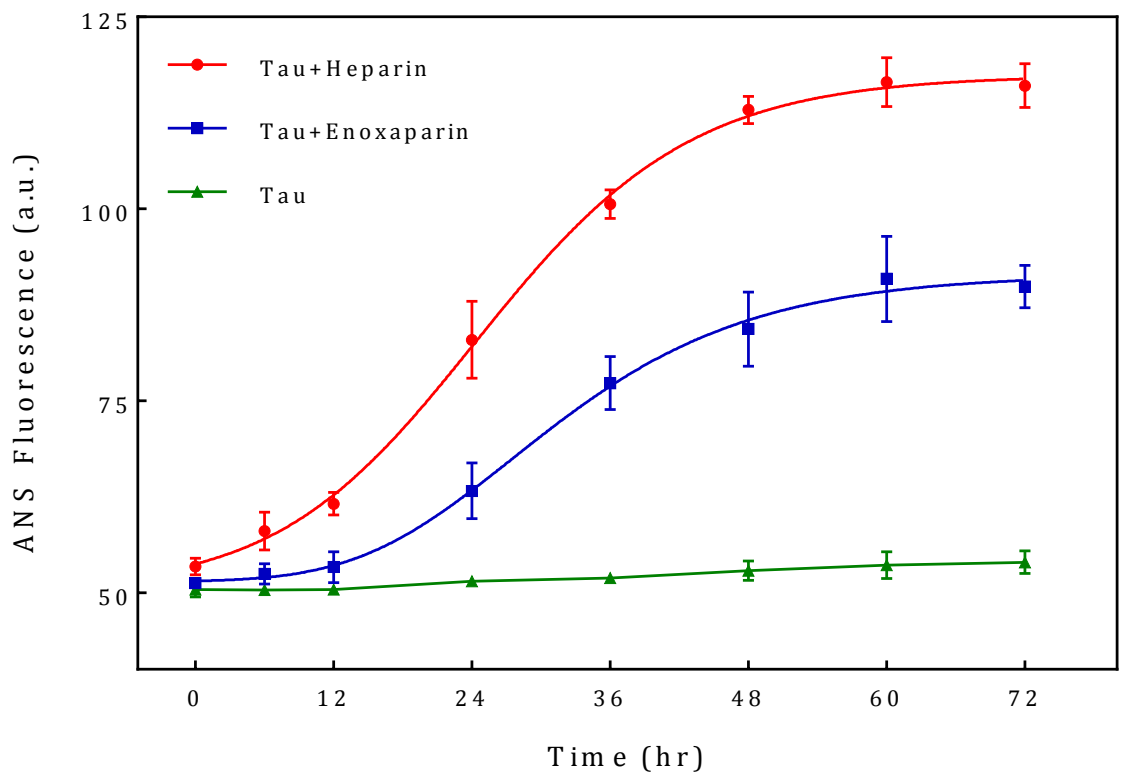

Fig. 8. Fibril formation of Tau monitored by the fluorescence of ANS. Aggregation was started with $58 \mu \mathrm{M}$ protein in the presence of $16 \mu \mathrm{m}$ heparin and $63 \mu \mathrm{m}$ enoxaparin incubated at $37^{\circ} \mathrm{C}$ in $50 \mathrm{mM}$ Tris- $\mathrm{HCl}$ buffer, $\mathrm{pH}$ 7.6, and $5 \mathrm{mM}$ DTT. At indicated Tau-inducer and ANS concentrations, the ANS fluorescence intensity measured in the presence of the heparin-induced intermediates was higher than that obtained with enoxaparin, indicating that the dye interacts with more nonpolar environment on the accessible surfaces of assembly-competent species. The error bars represent the spread in the data calculated from two or more independent experiments. 

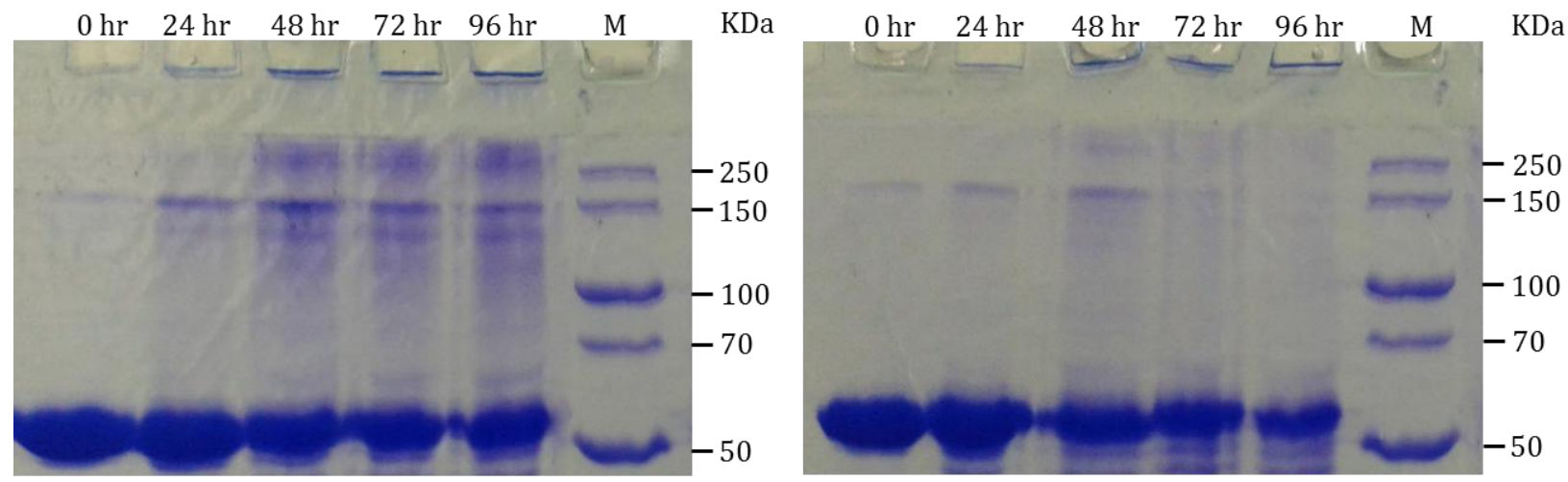

Fig. 9. SDS-PAGE analysis of Tau fibril formation. Tau was incubated for different periods of time $(0,24,48$, 72 and 96h) with16 $\mu \mathrm{m}$ heparin (Left) and $63 \mu \mathrm{m}$ enoxaparin (Right) and visualized by separating aggregation samples by 10\% SDS-PAGE, followed by protein staining with Coomassie Brilliant Blue. Incubation of Tau without heparin does not lead to formation of high molecular weight products (data not show). 

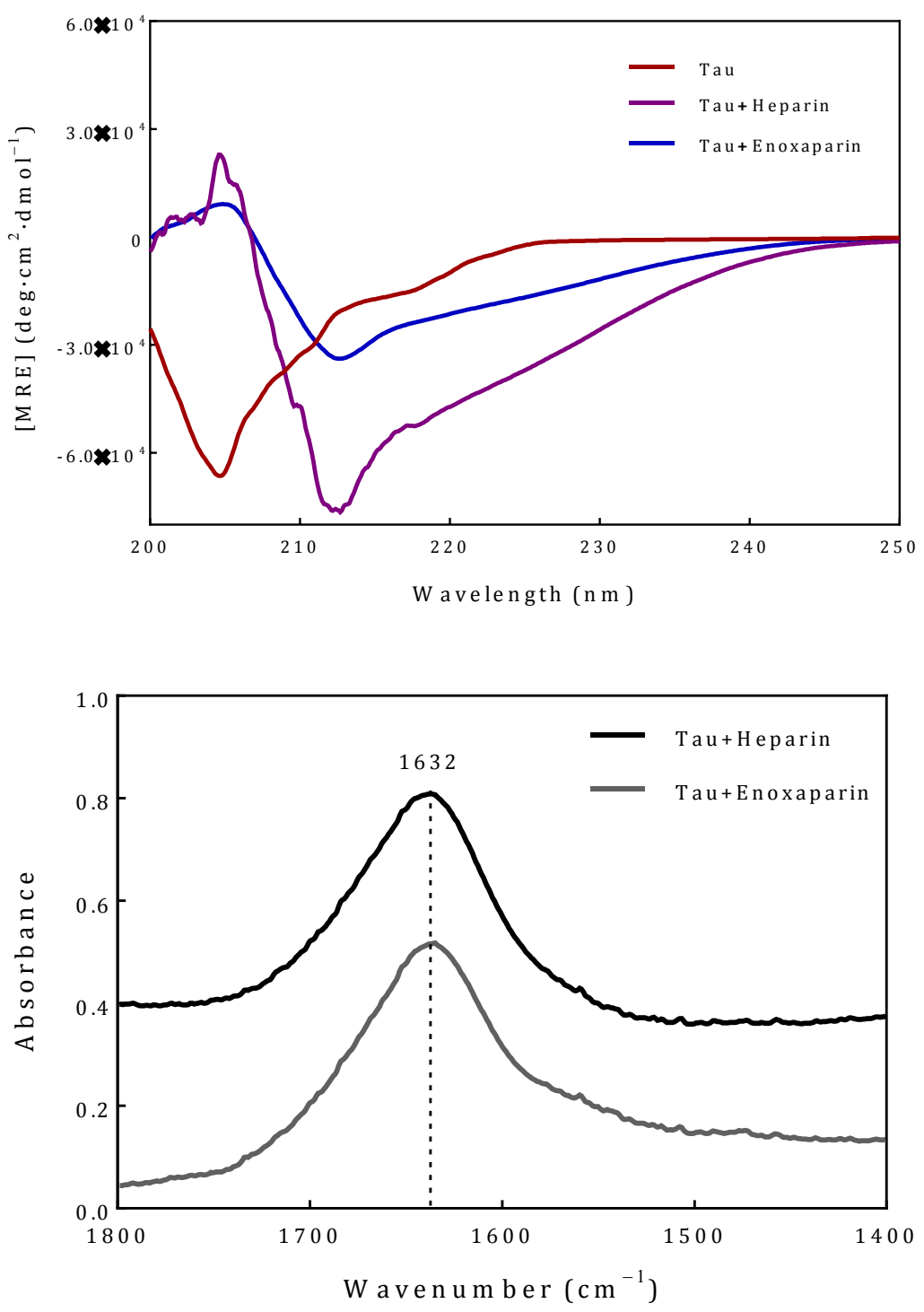

Fig. 10. (Top) Far UV CD spectra of control Tau protein and after fibril formation. Tau polymerization reactions at $58 \mu \mathrm{M}$ protein concentration were mixed with $16 \mu \mathrm{M}$ heparin and $63 \mu \mathrm{M}$ enoxaparin an incubated for $96 \mathrm{~h}$ at $37^{\circ} \mathrm{C}$. Each spectrum was obtained at $25{ }^{\circ} \mathrm{C}$ with a $1 \mathrm{~mm}$ path length cell and is the average of two scans. All necessary corrections have been made for background absorption. The analysis of soluble and polymerized Tau by CD shows a clear minimum for the spectrum of the soluble sample at $\sim 200$ $\mathrm{nm}$, indicating a mostly random coil structure, whereas the minimum is shifted towards higher wavelengths after polymerization, indicating an increase of $\beta$-structure. (Bottom) FTIR spectra of Tau fibril. Tau polymerization reactions at $58 \mu \mathrm{M}$ protein concentration were mixed with $16 \mu \mathrm{M}$ heparin and $63 \mu \mathrm{M}$ enoxaparin an incubated for $200 \mathrm{~h}$ at $37^{\circ} \mathrm{C}$. Comparing FTIR spectra confirms more increase of $\beta$-structure 
upon polymerization in the presence of heparin, due to the increase of absorbance at about $1632 \mathrm{~cm}^{-1}$ after polymerization.
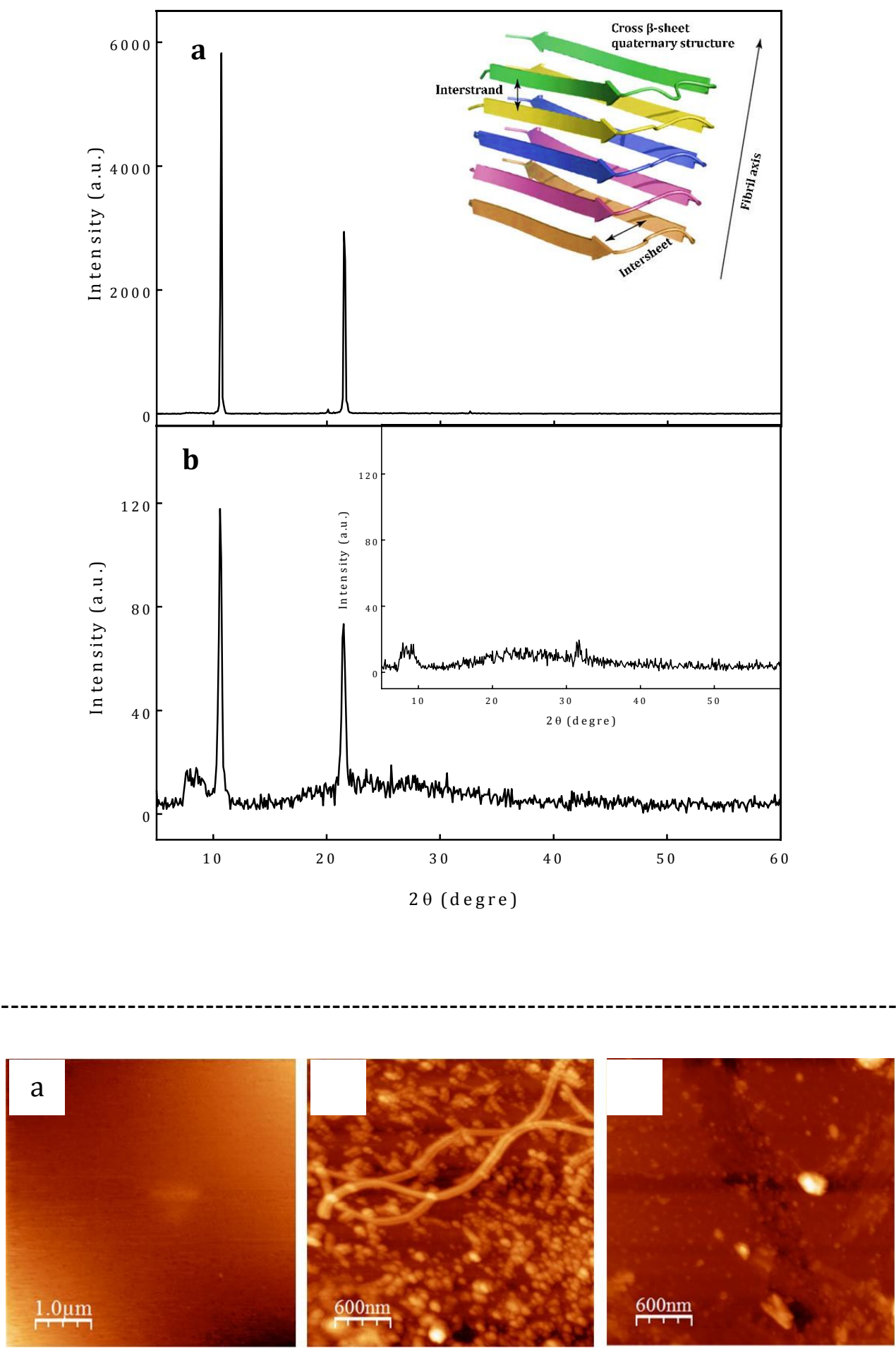
Fig. 11. (Top/Middle) The X-ray diffraction pattern of Tau with and without inducers were recorded after 96h incubated at $37{ }^{\circ} \mathrm{C}$ in $50 \mathrm{mM}$ Tris-HCl buffer, $\mathrm{pH}$ 7.6, and $5 \mathrm{mM}$ DTT using a Rigaku Miniflex X-ray diffractometer in $2 \theta$ ranging from $5^{\circ}$ to $60^{\circ}$. XRD pattern of Tau fibril formation in presence $16 \mu \mathrm{M}$ heparin (a) and $63 \mu \mathrm{M}$ enoxaparin (b) that exhibited two peaks at $2 \theta$ around of $10^{\circ}$ and $20^{\circ}$. X-ray diffractions of Tau fibrils induced by heparin/enoxaparin reveal the typical cross- $\beta$ diffraction pattern with a meridional reflection at $0.47 \mathrm{~nm}$ for the distance between two strands (denoted by peak of $2 \theta$ around $20^{\circ}$ ) within a $\beta$ sheet and a reflection at $1.07 \mathrm{~nm}$ on the equator (denoted by peak of $2 \theta$ around $10^{\circ}$ ) indicating the average distance of the inter-sheet stacking. The inset (a), for instance, shows $A \beta$ (1-42) fiber structure obtained from NMR that reveals the inter-strand and inter-sheet distances in cross- $\beta$ structure (PDB code: $2 B E G$ ) [65]. The inset (b) corresponds to native Tau as a control. (Bottom) Representative AFM images of Tau in the absence and presence of inducers. Tau incubated at $37^{\circ} \mathrm{C}$ for $120 \mathrm{~h}$ in the absence of inducers does not lead to fibril formation (a) but in the presence of heparin (b) and enoxaparin (c) form protein assembly. Note the presence of paired helical-like filaments in "b". 

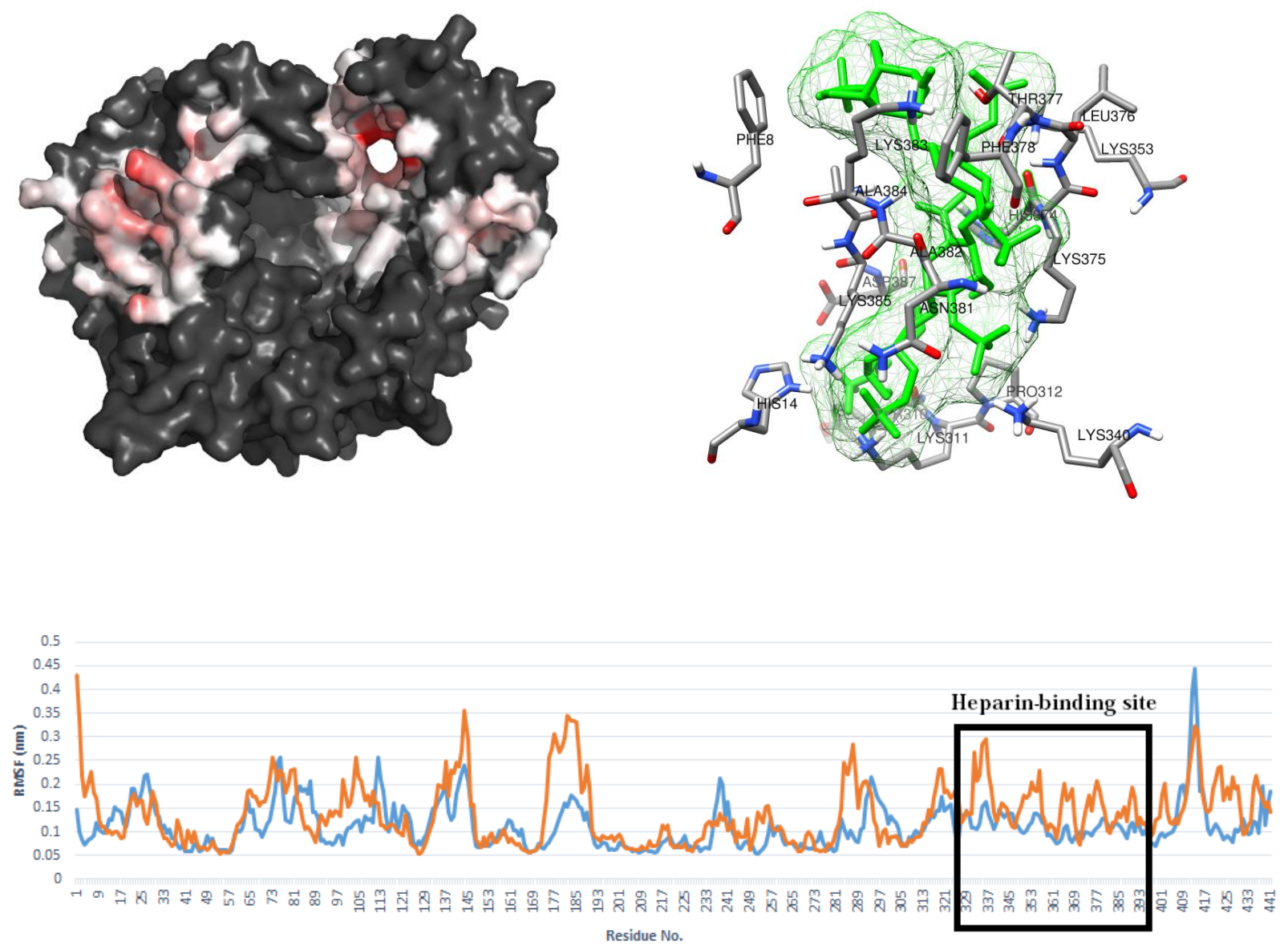

—Tau-heparin _Tau

Fig. 12. (Top) Heparin-binding sites (left) and the result of Tau-heparin docking (right). The heparin-binding sites determined by ClusPro are shown in lighter color. The heparin is shown in green color. (Bottom) Plotted RMSF values of Tau (orange) and Tau-heparin complex (blue). The main heparin-binding pocket of Tau is shown in an open box. 


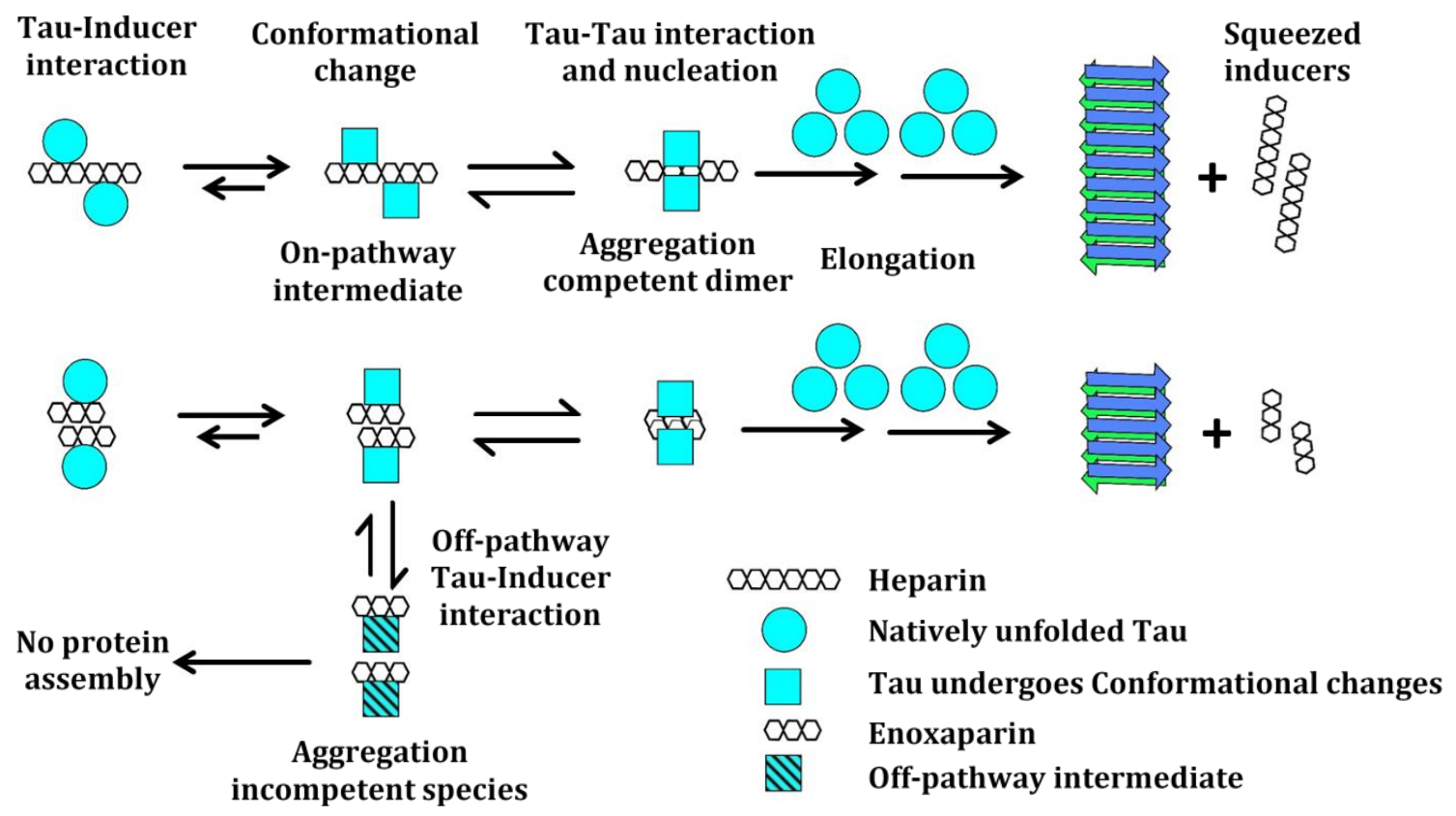

Scheme 1. Hypothetical comparative models for Tau amyloid formation in the presence of heparin and enoxaparin. Two Tau protein molecules bind each heparin simultaneously, undergo conformational changes (which is the rate-limiting step), then interact with each other through amyloidogenic segments ( $\mathrm{V}_{306} \mathrm{QIVYK}_{311}$ and $\mathrm{V}_{275} \mathrm{QIINK}{ }_{280}$ ). In presence of the shorter inducer, enoxaparin, (at $~ 1: 1$ stoichiometry), efficient Tau-Tau interaction and successful formation of on-pathway intermediates is relatively unlikely (compared to that of heparin). So step of fibril elongation occurs more successfully, in the presence of the longer inducer, by the process of monomer addition to protein-heparin-protein (PHP) ternary system, accompanied by PHP-PHP assembly, resulting in release of squeezed inducers. 